\title{
Estilos de aprendizaje: Un reto curricular para la educación en enfermería
}

\author{
Learning styles: a curricular challenge for nursing education \\ Estilos de aprendizagem: Um desafio curricular para a educação em enfermagem \\ Giselly Matagira-Rondón* \\ Martha Cecilia Torres-Panesso** \\ Natalia Berrio-Osorio ${ }^{* * *}$
}

\section{Resumen}

Objetivo: Determinar los estilos de aprendizaje y factores relacionados, en los estudiantes del programa de enfermería de una universidad privada en Medellín (Colombia). Materiales y métodos: Investigación transversal con intención analítica, aplicada a 179 estudiantes matriculados en el programa de enfermería. La recolección de los datos se realizó a través de: una encuesta autoaplicada que permitió indagar todo lo relacionado con variables sociodemográficas y personales; así como de un cuestionario CHAEA de Honey Alonso sobre estilos de aprendizaje (Activo, Reflexivo, Teórico y Pragmático). Las variables cualitativas se obtuvieron mediante medidas de frecuencia y las variables cuantitativas se consiguieron con medidas de tendencia central. Se realizó un análisis bivariado, utilizando la prueba X2 de Pearson; no se obtuvo significancia estadística ( $p>0,05)$. Resultados: En la población estudiada predominó con un $85,4 \%$ las mujeres; el estado civil fue el de soltero, con un $89 \%$. En cuanto a los recursos educativos empleados, se observó que: el $62,1 \%$ asiste a la biblioteca para búsqueda de información y el $65,9 \%$ se sirve de las bases de datos como fuentes de información. El método de aprendizaje más utilizado es el análisis de caso, aunque predomina en la enseñanza la magistralidad. El estilo de aprendizaje que predominó fue el reflexivo con un $15 \%$ seguido del teórico con un 14\%. Conclusiones: Los estudiantes de enfermería aplican los diferentes estilos de aprendizaje en los diversos semestres; sin embargo, existe una tendencia hacia el estilo de aprendizaje reflexivo y teórico; por tanto, conocer estas tendencias por los docentes universitarios es un reto, ya que se requiere emplear procedimientos diferentes a los habituales en la enseñanza y así desarrollar el pensamiento enfermero en los estudiantes, de acuerdo a su predilección de aprendizaje.

Palabras Clave: Aprendizaje, educación en enfermería, estudiantes de enfermería

\section{Abstract}

Objective: Determine the learning styles and related factors in students from the nursing program of a private university in Medellin (Colombia). Materials and Methods: Transversal research with analytical intention, applied to 179 students enrolled in the nursing program. The data was recollected through: a self-applied

\section{Autor de correspondencia}

* Enfermera. Magíster en Salud Pública. Docente Corporación Universitaria Adventista. Correo: gmatagira@unac.edu.co, Orcid: https://orcid.org/0000-0001-9642-1253 Medellín, Colombia.

** Enfermera. Magister en Enfermería. Docente Universidad de Antioquia. Correo: martha.torres@udea.edu.co, Orcid http:// orcid.org/0000-0002-7563-6965 Medellín, Colombia.

**** Enfermera. Especialista en Administración de Servicios de Salud. Docente Corporación Universitaria Adventista. Correo: nberrio@ unac.edu.co, Orcid http://orcid.org/00000002-7542-2027 Medellín, Colombia. 
ISSN-PRINT

1794-9831

E-ISSN 2322-7028

Vol. 16 No. 2

May - Ago 2019

Cúcuta, Colombia interview that allowed to investigate relating sociodemographic and personal variables; as well as a questionnaire of Honey Alonso, CHAEA, about learning styles (Active, Reflexive, Theoretical and Pragmatic). The qualitative variables were obtained through frequency measures and the quantitative variables were obtained through central tendency. A bivariate analysis was performed using the Pearson's chi-squared test; no statistical significance was obtained ( $>>0,05)$. Results: In the population studied women predominated with $85.4 \%$; and the marital status 'single' with $89 \%$. Regarding educative resources used, it was observed that: $62.1 \%$ go to the library to find information, and $65.9 \%$ use data bases as sources of information. The most used learning style was reflexive with $15 \%$ followed by theoretical with 14\%. Conclusions: Nursing students apply the different learning styles in diverse semesters; however, there is a tendency toward the reflexive and theoretical styles; therefore, knowing these tendencies is a challenge for university teachers, since it requires performing different learning procedures to develop the nursing thinking in the students, according to their learning preference.

Keywords: Learning, Nursing Education, Nursing Students.

\section{Resumo}

Objetivo: Determinar os estilos de aprendizagem e fatores relacionados, nos alunos de uma escola de enfermagem de uma universidade particular de Medellin (Colômbia). Materiais e métodos: Estudo de tipo transversal de intenção analítica aplicado em 179 alunos da enfermagem. Os dados foram coletados aferindo variáveis sociais, demográficas e pessoais; também foi empregado o questionário CHAEA de Honey-Alonso sobre os estilos de aprendizagem (Ativo, Reflexivo, Teórico e Pragmático). As variáveis qualitativas foram apresentadas com medidas de frequência e as variáveis quantitativas apresentaram-se com medidas de tendência central. Foi feita uma análise bivariada empregando o teste X2 de Pearson sem obter significância estatística ( $p>0,05)$. Resultados: O 85,4\% da população estudada foi do sexo feminino de estado civil solteiro (89\%). Nos recursos de ensino empregados, o $62,1 \%$ frequenta a biblioteca para pesquisar informação e o $65.9 \%$ usa portais de pesquisa acadêmica. $\mathrm{O}$ método de aprendizagem mais usado é a análise de casos, embora o que predomina são as aulas magistrais. O estilo de aprendizagem mais frequente foi o reflexivo (15\%), seguido do teórico com um 14\%. Conclusões: Os alunos da enfermagem usam os diferentes estilos de aprendizagem durante a sua formação. Embora exista uma tendência ao aprendizagem reflexivo e teórico, conhecer às tendências dos estilos de aprendizagem dos alunos pelos professores é um desafio, já que precisa de métodos de ensino deferentes dos habituais para assim desenvolver o pensamento enfermeiro, acorde com as preferencias do aprendizagem.

Palavras-chave: Aprendizagem, educação em enfermagem, estudantes de enfermagem

\section{Introducción}

En la actualidad, de acuerdo con González et al, (1) el proceso de enseñanza aprendizaje, requiere que el sector educativo esté en capacidad de formar profesionales de excelencia, competitivos, que logren adaptarse a los cambios y exigencias de la sociedad, lo que implica grandes y permanentes desafíos a nivel del diseño de los programas académicos. Como lo señalan González y Maciá (2), la formación de estudiantes de enfermería, debe asegurar que los profesionales ofrezcan cuidados seguros bajo normas de calidad, lo que implica que todos los actores del proceso enseñanza- aprendizaje, (estudiantes, profesores, tutores) estén en sinergia para lograrlo.

Por consiguiente, de acuerdo a las condiciones que presente dicho proceso, según lo mencionado por González et al. (1), el rendimiento académico en los estudiantes, puede verse afectado por determinantes personales, sociales e institucionales. Dentro de los personales se evidencian las condiciones cognitivas como: la motivación, las estrategias de aprendizaje, el auto concepto académico, la asistencia a clases, la inteligencia y aptitudes. En los determinantes sociales cobran importancia el entorno familiar, el nivel educativo de los padres, las variables demográficas y el contexto socioeconómico. De otra parte, los determinantes institucionales están en relación directa con la elección de estudios según los intereses del estudiante, la complejidad de los temas, la relación estudiante-profesor, el ambiente estudiantil y los servicios institucionales, entre otros. 
En contraste con lo anterior, es necesario resaltar que cuando ingresan los estudiantes provenientes de la educación media al ámbito universitario, se espera que tengan un desarrollo avanzado en hábitos de estudio, que hayan desarrollado y aplicado un estilo de aprendizaje, producto de unas estrategias que lo conduzcan hacia el logro de unos excelentes resultados académicos. Pero lo que sucede en realidad, según Isaza (3) es que, en la universidad, se identifican estudiantes con bajas posibilidades académicas que no les permite progresar y dar respuesta a las demandas del nuevo proceso de aprendizaje. Este bajo rendimiento, frecuentemente es asociado a los métodos y estrategias que utilizan para aprender y que en ocasiones no están articulados con la forma de enseñar de los docentes de educación superior.

En términos de Gordón (4) se resalta que el desconocimiento de los estilos de aprendizaje por parte de los docentes y de los estudiantes, puede ocasionar disfunciones cognitivas que impiden desarrollar todo el potencial que se podría lograr; mientras que entenderlos, según señalan Bayona y Campo (5), ayuda a que se tengan en cuenta una variedad de estrategias de enseñanza, metodología y evaluación que logren identificar los aspectos necesarios a fortalecer en el proceso de aprendizaje.

En este sentido, Yancen et al. (6), plantean que la importancia del emparejamiento entre el estilo de aprendizaje por parte de los alumnos y el estilo de enseñar por parte de los docentes, será adecuado si van hacia la misma dirección del rendimiento en alguna materia, aunque el desemparejamiento permitiría el desarrollo del pensamiento creativo; es necesario, por tanto, conocer cómo aprenden los estudiantes para mejorar la efectividad y la eficacia de los programas de formación y desarrollo.

También es importante tener en cuenta como lo afirman González et al. (1), que los estudiantes de enfermería deben desarrollar el pensamiento crítico y adquirir habilidades para tomar decisiones responsables sobre la resolución de problemas para el buen desempeño en el campo profesional. El desarrollo del pensamiento crítico en el proceso de aprendizaje implica que el estudiante sea capaz de cuestionar la información, realizar análisis profundos con lógica e imparcialidad, comunicar a través de expresiones orales y escritas sus ideas; es decir, que sea capaz de innovar en la formulación de proyectos y que tome decisiones que contribuyan al bienestar social con responsabilidad, actuando bajo un marco ético y valórico. La adquisición de estas habilidades es responsabilidad de los docentes quienes deben proponer estrategias para llevar a cabo estas competencias en los estudiantes.

Por otra parte, Gordon (4) sugiere que definir el estilo de aprendizaje de los estudiantes permite generar mecanismos para lograr un aprendizaje significativo, es por ello que dichos estilos se reconocen en la educación superior como herramientas que permite a los docentes orientar acciones pedagógicas coherentes con las necesidades y capacidades de los alumnos. Bajo este contexto radicó la importancia del presente estudio, el cual, mediante la aplicación del cuestionario Honey -Alonso de estilos de aprendizaje CHAEA, logró identificar los estilos de aprendizaje de los estudiantes del programa de enfermería, teniendo en cuenta las competencias básicas y necesarias como el desarrollo del pensamiento crítico y la toma de decisiones éticas y responsables que se requieren durante su formación y posterior ejercicio profesional.

Sumado a lo anterior, en Colombia se encuentran algunas investigaciones relacionadas con estilos de aprendizaje en otras áreas y poco se ha investigado sobre el tema en el área de enfermería, por tanto, conocer y entender los estilos activo, reflexivo, teórico y pragmático aplicados al componente disciplinar, se concibe como una pieza clave que conlleva al éxito del proceso enseñanza-aprendizaje ajustando el currículo a las necesidades de los estudiantes.

\section{Objetivos}

\section{Objetivo general}

Determinar los estilos de aprendizaje y sus factores relacionados, en estudiantes del programa de enfermería de una universidad de Medellín, con el propósito de orientar la formulación de métodos de enseñanza aprendizaje, acorde a las necesidades de los estudiantes y el currículo.

\section{Objetivos específicos}

- Caracterizar los factores sociodemográficos, nivel educativo de los padres o tutores, motivo para estudiar enfermería y los recursos educativos, presentes en los estudiantes del programa de enfermería.
E-ISSN 2322-7028

Vol. 16 No. 2

May - Ago 2019

Cúcuta, Colombia 
ISSN-PRINT

1794-9831

E-ISSN 2322-7028

Vol. 16 No. 2

May - Ago 2019

Cúcuta, Colombia
- Establecer cuáles son los estilos de aprendizaje de los estudiantes del programa de enfermería.

- Identificar la asociación entre los factores sociodemográficos, personales y de recursos educativos de los estudiantes y los estilos de aprendizaje.

\section{Materiales y métodos}

Se realizó un estudio transversal con intención analítica, en el primer período académico del año 2017. No fue necesario calcular muestra teniendo en cuenta que se convocó a todos los estudiantes matriculados en el programa de Enfermería del primer al décimo semestre en la sala de cómputo de la universidad. Para cada semestre se reservó un día respectivamente para aplicar el cuestionario; participaron voluntariamente 179 estudiantes, los que no participaron se debió a que no asistieron a la institución el día que tuvieron asignado por semestre.

Para la recolección de los datos se realizó una encuesta autoaplicada que incluyó variables sociodemográficas y personales en donde se indagó: si labora actualmente, área en la que labora y horas de trabajo a la semana, motivación para estudiar enfermería y si tenía otro tipo de estudio técnico o universitario. Dentro de las variables para identificar el empleo de recursos educativos se evaluó el uso de las TICS por parte de los estudiantes, de las bases de datos y métodos utilizados en el proceso enseñanza aprendizaje que más animan al estudiante a aprender.

Se aplicó el cuestionario CHAEA de Honey Alonso de estilos de aprendizaje, el cual, ha sido utilizado de forma amplia en estudios investigativos en diversos países de habla Hispana como: España, Perú, Argentina y Venezuela entre otros, en donde se reportó confiabilidad del total del instrumento con un alpha de cronbach del 0.789 (7). En Colombia el cuestionario fue validado por expertos (3) y usado en trabajos similares $(8,9)$. Presenta una relación de 80 ítems sobre Estilos de Aprendizaje a los que es necesario responder $+\mathrm{o}-$-. Los ítems se estructuran en cuatro grupos o secciones de 20 ítems correspondientes a los cuatro Estilos de Aprendizaje: Activo, reflexivo, teórico y pragmático. Todos los ítems están distribuidos aleatoriamente formando un sólo conjunto. La puntuación absoluta que el sujeto obtiene en cada grupo de 20 ítems, será el nivel que alcance en cada uno de los cuatro Estilos de Aprendizaje (10).
El instrumento fue sometido a una prueba piloto, con un grupo de estudiantes de Primer Semestre donde participaron 15 estudiantes; posterior a la realización de la prueba se verificó el tiempo requerido para contestar el instrumento, la facilidad o no del diligenciamiento electrónico y se modificó la estructura de preguntas y respuestas, agrupándolas de tal manera que fuera más fácil el proceso.

Los datos se analizaron en el paquete estadístico SPSS versión 21, se realizó un análisis univariado de las variables sociodemográficas, personales, educativas y los estilos de aprendizaje. Las variables cualitativas se presentaron en medidas de frecuencia y las cuantitativas se presentaron con medidas de tendencia central. Se realizó un análisis bivariado utilizando la prueba de X2 de Pearson, no se obtuvo significancia estadística $(\mathrm{p}>0,05)$.

El presente trabajo de investigación se ajustó a las recomendaciones éticas dadas por la declaración de Helsinki para investigación en seres humanos; por las características del trabajo, su metodología y teniendo en cuenta el artículo 11 de la resolución 8430 de 1993, se clasificó como una investigación de riesgo mínimo, ya que los datos se obtendrán a través de encuesta sin ningún tipo de intervención que ponga en riesgo la integridad física o mental de los estudiantes.

Los participantes lo hicieron voluntariamente firmando el consentimiento informado, previa explicación de los objetivos y fines del estudio. Se tuvo el aval del comité de ética de la universidad como lo certifica la resolución 001/17.

\section{Resultados}

\section{Características sociodemográficas y personales:}

De 233 estudiantes de enfermería matriculados en el primer período académico del año 2017, participaron 179 (76,8\%). La mayor participación fue en el cuarto semestre con 31 (17,3\%), seguido de segundo y quinto semestre 26 y $21(14,5 \%, 11.7 \%)$ respectivamente, sexto $19(10.6 \%)$, primero y décimo con 18 estudiantes $(10,1 \%)$, en tercer, octavo y séptimo semestre participaron 17,12 y 9 estudiantes $(9,5 \%$, $6,7 \%, 5 \%$ ), la menor participación fue de noveno semestre $8(4,5 \%)$. La mediana para la edad fue de 22 años, rango intercuartil 26, la persona más joven tenía 17 años y la mayor 43. El 85,4\% eran mujeres, el estado civil predominante fue soltero en $89 \%$. La 
mayoría provienen de municipios (49,7\%). En cuanto al nivel educativo de los padres o tutores se encontró que el nivel técnico es el más frecuente en un $27 \%$

y el principal motivo para estudiar enfermería fue el deseo de ser enfermero con un 66\% (Tabla 1).

Tabla 1: Distribución porcentual de las características sociodemográficas, nivel educativo de los padres o tutores y motivo para estudiar enfermería de los estudiantes participantes del estudio, 2017

\begin{tabular}{|c|c|c|c|}
\hline Variable & Categoría & $\mathrm{n}$ & $\%$ \\
\hline \multirow{2}{*}{ Sexo } & Mujer & 153 & 85,4 \\
\hline & Hombre & 26 & 14,6 \\
\hline \multirow{4}{*}{ Estado civil } & Soltero & 159 & 88,8 \\
\hline & Casado & 13 & 7,3 \\
\hline & Separado - divorciado & 1 & 0,6 \\
\hline & Unión libre & 6 & 3,4 \\
\hline \multirow{3}{*}{ Procedencia } & Capital de departamento & 84 & 46,9 \\
\hline & Municipio & 89 & 49,7 \\
\hline & Vereda & 6 & 3,4 \\
\hline \multirow{6}{*}{ Nivel educativo de la madre o tutora } & Primaria & 32 & 17,9 \\
\hline & Secundaria & 57 & 31,8 \\
\hline & Técnica & 49 & 27,4 \\
\hline & Universitaria & 28 & 15,6 \\
\hline & Especialización & 12 & 6,7 \\
\hline & Ninguno & 1 & 0,6 \\
\hline \multirow{9}{*}{ Nivel educativo del padre o tutor } & Primaria & 41 & 22,9 \\
\hline & Secundaria & 47 & 26,3 \\
\hline & Técnica & 27 & 15,1 \\
\hline & Universitaria & 39 & 21,8 \\
\hline & Especialización & 16 & 8,9 \\
\hline & Ninguno & 2 & 1,1 \\
\hline & No se & 7 & 3,9 \\
\hline & $37-48$ & 15 & 8,4 \\
\hline & No trabajo & 137 & 76,5 \\
\hline \multirow{6}{*}{ Motivo estudiar enfermería } & El programa académico & 17 & 9,5 \\
\hline & Mejorar los conocimientos previos & 37 & 20,7 \\
\hline & Mi sueño fue ser siempre enfermero & 118 & 65,9 \\
\hline & Presión de los padres o tutores & 3 & 1,7 \\
\hline & No tuve otra opción & 4 & 2,2 \\
\hline & Ninguna & 84 & 46,9 \\
\hline
\end{tabular}

Fuente: Base de datos de las autoras

\section{Recursos educativos}

En cuanto a los recursos educativos, según se observa en la tabla 2 , el $62,1 \%$ refiere que asiste a la biblioteca para realizar búsqueda de información, el 31,8\% utiliza libros de la biblioteca virtual y el $48 \%$ usa las TICS algunas veces, mientras que solo el $20,1 \%$ las utiliza siempre; a su vez, las bases de datos son em- pleadas como fuente de información por el $65,9 \%$ de los estudiantes. Dentro de los métodos didácticos usados por los docentes el $62 \%$ señala que el análisis de casos son los que más los animan a aprender, aunque no es el método más utilizado por los docentes en el momento de la enseñanza en el aula, siendo predominante la clase magistral con un $63.1 \%$. 
ISSN-PRINT

1794-9831

E-ISSN 2322-7028

Vol. 16 No. 2

May - Ago 2019

Cúcuta, Colombia

Tabla 2: Distribución porcentual de las variables relacionadas con recursos educativos en los encuestados (2017)

\begin{tabular}{|c|c|c|c|}
\hline Variable & Categoría & $\mathrm{n}$ & $\%$ \\
\hline \multirow{2}{*}{ Uso de la biblioteca física } & $\mathrm{Si}$ & 112 & 62,1 \\
\hline & No & 67 & 37,4 \\
\hline \multirow{2}{*}{ Uso de la biblioteca virtual } & $\mathrm{Si}$ & 57 & 31,8 \\
\hline & No & 122 & 68,2 \\
\hline \multirow{2}{*}{ Uso de bases de datos } & $\mathrm{Si}$ & 118 & 65,9 \\
\hline & No & 61 & 34,1 \\
\hline \multirow{4}{*}{ Uso de las TICS } & Siempre & 36 & 20,1 \\
\hline & Casi siempre & 52 & 29,1 \\
\hline & Algunas veces & 86 & 48 \\
\hline & Nunca & 5 & 2,8 \\
\hline \multirow{4}{*}{$\begin{array}{l}\text { Métodos usados por los docentes en clases teó- } \\
\text { ricas }\end{array}$} & Clase magistral & 113 & 63,1 \\
\hline & Análisis de casos & 12 & 6,7 \\
\hline & Seminarios participativos & 38 & 21,2 \\
\hline & Socialización de guías de trabajo autónomo & 16 & 8,9 \\
\hline \multirow{5}{*}{ Métodos que motivan más el aprendizaje } & Clase magistral & 28 & 15,6 \\
\hline & Análisis de casos & 62 & 34,6 \\
\hline & Seminarios participativos & 57 & 31,8 \\
\hline & Socialización de guías de trabajo autónomo & 26 & 14,5 \\
\hline & Otros & 6 & 3,4 \\
\hline
\end{tabular}

Fuente: Base de datos de las autoras

Estilos de aprendizaje según cuestionario CHAEA Honey Alonso

El estilo de aprendizaje que predominó fue el reflexivo en $15 \%$ con una desviación típica de 2,5 seguido del teórico 14\% con una desviación típica de 2,7; en tercer lugar, el pragmático en un $12,6 \%$ con una desviación típica de 2,7 y por último, el activo en un $10,4 \%$ con una desviación típica de 2,9 .

Dentro de los estilos de aprendizaje que más prevalecieron fueron el reflexivo y el teórico; la categoría más frecuente fue la moderada en un $64 \%$ y $37 \%$ respectivamente como se observa en la figura 1 y 2 .

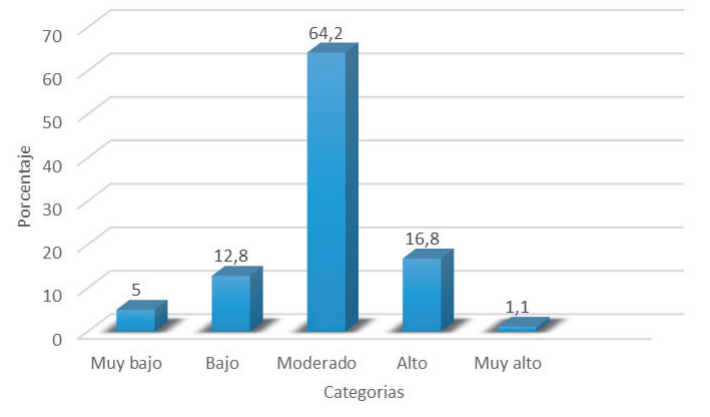

Figura 1: Distribución porcentual de las preferencias del estilo de aprendizaje Reflexivo en los participantes del estudio (2017).

Fuente: Base de datos estilos de aprendizaje elaboración de las autoras. 


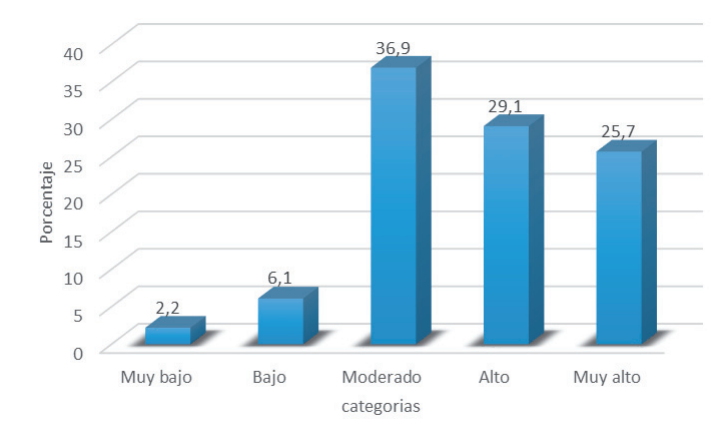

$\overline{\text { E-ISSN } 2322-7028}$

Vol. 16 No. 2

May - Ago 2019

Cúcuta, Colombia

Figura 2: Distribución porcentual de las preferencias del estilo de aprendizaje Teórico en los participantes del estudio (2017).

Fuente: Base de datos estilos de aprendizaje elaboración de las autoras.

Como se observa en la tabla 3, el estilo de aprendizaje más frecuente en los estudiantes del primer semestre fue el teórico con un $89 \%$, seguido del reflexivo con un $83,3 \%$. Para el segundo semestre el más frecuente fue el estilo activo con $96,2 \%$. El tercer semestre tuvo dos estilos ponderados de igual manera, fueron el reflexivo y el pragmático con un $88,2 \%$. Dentro del cuarto semestre el estilo predominante fue el teórico con $90,3 \%$.
Para el quinto y el sexto semestre el estilo teórico fue el que tuvo mayor frecuencia, siendo $95,2 \%$ y $100 \%$ respectivamente. Para el séptimo semestre el estilo reflexivo fue el que más predominó con un $100 \%$ y para el octavo semestre el teórico en un $92 \%$. Noveno semestre tuvo los estilos activo, reflexivo y teórico ponderados de igual manera en un $87,5 \%$ respectivamente y para décimo semestre el mayor porcentaje se presentó en el estilo teórico.

Tabla 3: Distribución porcentual de estilos de aprendizaje por semestre académico (2017)

\begin{tabular}{ccccc}
\hline Semestre & \multicolumn{4}{c}{ Estilo de Aprendizaje } \\
& Activo & Reflexivo & Teórico & Pragmático \\
\hline 1 & $66,70 \%$ & $83,30 \%$ & $\mathbf{8 8 , 9 0 \%}$ & $66,70 \%$ \\
\hline 2 & $\mathbf{9 6 , 2 0 \%}$ & $76,90 \%$ & $88,50 \%$ & $84,60 \%$ \\
\hline 3 & $64,70 \%$ & $\mathbf{8 8 , 2 0 \%}$ & $100,00 \%$ & $\mathbf{8 8 , 2 0 \%}$ \\
\hline 4 & $74,20 \%$ & $77,40 \%$ & $\mathbf{9 0 , 3 0 \%}$ & $83,90 \%$ \\
\hline 5 & $90,50 \%$ & $90,50 \%$ & $\mathbf{9 5 , 2 0 \%}$ & $\mathbf{9 5 , 2 0 \%}$ \\
\hline 6 & $63,20 \%$ & $78,90 \%$ & $\mathbf{1 0 0 , 0 0 \%}$ & $73,70 \%$ \\
\hline 7 & $77,80 \%$ & $\mathbf{1 0 0 , 0 0 \%}$ & $88,90 \%$ & $55,60 \%$ \\
\hline 8 & $58,30 \%$ & $75,00 \%$ & $\mathbf{9 1 , 7 0 \%}$ & $83,30 \%$ \\
\hline 9 & $\mathbf{8 7 , 5 0 \%}$ & $\mathbf{8 7 , 5 0 \%}$ & $\mathbf{8 7 , 5 0 \%}$ & $75,00 \%$ \\
\hline 10 & $66,70 \%$ & $77,80 \%$ & $\mathbf{8 3 , 3 0 \%}$ & $66,70 \%$ \\
\hline
\end{tabular}

Fuente: Base de datos elaboración por las autoras

Aunque el estudio en principio pretendía analizar la correlación entre los factores sociodemográficos, nivel educativo de los padres o tutores, motivo para estudiar enfermería y los recursos educativos con los estilos de aprendizaje de los estudiantes, no se encontró significancia estadística: X2 de Pearson, ( $p$ > 0,05) (Tabla 4). 
ISSN-PRINT

1794-9831

E-ISSN 2322-7028

Vol. 16 No. 2

May - Ago 2019

Cúcuta, Colombia
Tabla 4: Análisis bivariado de los estilos de aprendizaje y su asociación con variables sociodemográficas, nivel educativo de los padres o tutores, motivo para estudiar enfermería y los recursos educativos, estudiantes de enfermería (2017).

\begin{tabular}{|c|c|c|c|c|c|c|c|c|c|c|c|c|c|}
\hline Variable & Categoría & $\begin{array}{l}\text { Estilo ap } \\
\text { zaje Ref } \\
\text { Alto - } \\
\text { Mode- } \\
\text { rado }\end{array}$ & $\begin{array}{l}\text { rendi- } \\
\text { exivo }\end{array}$ & $\begin{array}{l}\text { Chi Cuadra- } \\
\text { do/ Valor P }\end{array}$ & $\begin{array}{c}\text { Estilo a } \\
\text { dizaje T } \\
\text { Alto - } \\
\text { Mode- } \\
\text { rado }\end{array}$ & $\begin{array}{l}\text { pren- } \\
\text { 'eórico } \\
\text { Bajo }\end{array}$ & $\begin{array}{c}\text { Chi } \\
\text { Cuadrado/ } \\
\text { Valor P }\end{array}$ & $\begin{array}{l}\text { Estilo a } \\
\text { dizaje A } \\
\text { Alto - } \\
\text { Mode- } \\
\text { rado }\end{array}$ & $\begin{array}{l}\text { pren- } \\
\text { Activo } \\
\text { Bajo }\end{array}$ & $\begin{array}{c}\text { Chi } \\
\text { Cuadrado/ } \\
\text { Valor P }\end{array}$ & $\begin{array}{r}\text { Estilo ap } \\
\text { dizaje } \mathrm{I} \\
\text { máti } \\
\text { Alto - } \\
\text { Mode- } \\
\text { rado }\end{array}$ & $\begin{array}{l}\text { pren- } \\
\text { Prag- } \\
\text { co }\end{array}$ & $\begin{array}{c}\text { Chi } \\
\text { Cuadrado/ } \\
\text { Valor P }\end{array}$ \\
\hline \multirow{4}{*}{ Estado civil } & Soltero & 129 & 30 & \multirow{4}{*}{$1,249 / 0,741$} & 145 & 14 & \multirow{4}{*}{$0,686 / 0,877$} & 120 & 39 & \multirow{4}{*}{$3,287 / 0,349$} & 125 & 34 & \multirow{4}{*}{$1,919 / 0,589$} \\
\hline & Casado & 12 & 1 & & 12 & 1 & & 10 & 3 & & 10 & 3 & \\
\hline & $\begin{array}{l}\text { Separado- } \\
\text { divorciado }\end{array}$ & 1 & 0 & & 1 & 0 & & 0 & 1 & & 1 & 0 & \\
\hline & Unión libre & 5 & 1 & & 6 & 0 & & 5 & 1 & & 6 & 0 & \\
\hline \multirow{3}{*}{ Procedencia } & Capital & 67 & 17 & \multirow{3}{*}{$1,944 / 0,378$} & 78 & 6 & \multirow{3}{*}{$1,064 / 0,587$} & 69 & 15 & \multirow{3}{*}{$3,887 / 0,143$} & 67 & 17 & \multirow{3}{*}{$0,093 / 0,954$} \\
\hline & Municipio & 76 & 13 & & 80 & 9 & & 62 & 27 & & 70 & 19 & \\
\hline & Vereda & 4 & 2 & & 6 & 0 & & 4 & 2 & & 5 & 1 & \\
\hline \multirow{6}{*}{$\begin{array}{c}\text { Nivel } \\
\text { educativo } \\
\text { de la madre } \\
\text { o tutora }\end{array}$} & Primaria & 27 & 5 & \multirow{6}{*}{$6,316 / 0,277$} & 30 & 2 & \multirow{6}{*}{$0,494 / 0,992$} & 23 & 9 & \multirow{6}{*}{$7,842 / 0,165$} & 24 & 8 & \multirow{6}{*}{$4,027 / 0,545$} \\
\hline & Secundaria & 48 & 9 & & 52 & 5 & & 42 & 15 & & 44 & 13 & \\
\hline & Técnica & 39 & 10 & & 45 & 4 & & 38 & 11 & & 37 & 12 & \\
\hline & $\begin{array}{l}\text { Universi- } \\
\text { taria }\end{array}$ & 25 & 3 & & 25 & 3 & & 25 & 3 & & 25 & 3 & \\
\hline & $\begin{array}{l}\text { Especializa- } \\
\text { ción }\end{array}$ & 7 & 5 & & 11 & 1 & & 6 & 6 & & 11 & 1 & \\
\hline & Ninguno & 1 & 0 & & 1 & 0 & & 1 & 0 & & 1 & 0 & \\
\hline \multirow{7}{*}{$\begin{array}{c}\text { Nivel } \\
\text { educativo } \\
\text { del padre o } \\
\text { tutor }\end{array}$} & Primaria & 35 & 6 & \multirow{7}{*}{$1,886 / 0,930$} & 39 & 2 & \multirow{7}{*}{$2,485 / 0,870$} & 29 & 12 & \multirow{7}{*}{$1,805 / 0,937$} & 36 & 5 & \multirow{7}{*}{$6,605 / 0,359$} \\
\hline & Secundaria & 37 & 10 & & 41 & 6 & & 35 & 12 & & 34 & 13 & \\
\hline & Técnica & 23 & 4 & & 25 & 2 & & 22 & 5 & & 20 & 7 & \\
\hline & $\begin{array}{l}\text { Universi- } \\
\text { taria }\end{array}$ & 32 & 7 & & 36 & 3 & & 30 & 9 & & 30 & 9 & \\
\hline & $\begin{array}{l}\text { Especializa- } \\
\text { ción }\end{array}$ & 12 & 4 & & 15 & 1 & & 12 & 4 & & 15 & 1 & \\
\hline & Ninguno & 2 & 0 & & 2 & 0 & & 2 & 0 & & 2 & 0 & \\
\hline & No se & 6 & 1 & & 6 & 1 & & 5 & 2 & & 5 & 2 & \\
\hline \multirow{5}{*}{$\begin{array}{c}\text { Motivo } \\
\text { estudiar } \\
\text { enfermería }\end{array}$} & $\begin{array}{l}\text { El programa } \\
\text { académico }\end{array}$ & 13 & 4 & \multirow{5}{*}{$3,197 / 0,525$} & 15 & 2 & \multirow{5}{*}{$4,742 / 0,315$} & 11 & 5 & \multirow{5}{*}{$12,710 / 0,13$} & 14 & 3 & \multirow{5}{*}{$5,096 / 0,278$} \\
\hline & $\begin{array}{l}\text { Mejorar } \\
\text { los cono- } \\
\text { cimientos } \\
\text { previos }\end{array}$ & 33 & 4 & & 36 & 1 & & 22 & 15 & & 30 & 7 & \\
\hline & $\begin{array}{l}\text { Mi sueño } \\
\text { fue ser } \\
\text { siempre } \\
\text { enfermero }\end{array}$ & 95 & 23 & & 107 & 11 & & 98 & 20 & & 93 & 25 & \\
\hline & $\begin{array}{l}\text { Presión de } \\
\text { los padres o } \\
\text { tutores }\end{array}$ & 2 & 1 & & 2 & 1 & & 1 & 2 & & 1 & 2 & \\
\hline & $\begin{array}{l}\text { No tuve } \\
\text { otra opción }\end{array}$ & 4 & 0 & & 4 & 0 & & 3 & 1 & & 4 & 0 & \\
\hline \multirow{2}{*}{$\begin{array}{l}\text { Uso de la } \\
\text { biblioteca } \\
\text { física }\end{array}$} & $\mathrm{Si}$ & 92 & 20 & & 101 & 11 & $0810 / 0368$ & 88 & 24 & $1,64 / 0205$ & 91 & 21 & $0673 / 0412$ \\
\hline & No & 55 & 12 & $0,000 / 0,993$ & 63 & 4 & $0,810 / 0,368$ & 47 & 20 & $1,04 / 0,205$ & 51 & 16 & $0,0 / 3 / 0,412$ \\
\hline $\begin{array}{l}\text { Uso de la } \\
\text { biblioteca }\end{array}$ & $\mathrm{Si}$ & 49 & 8 & $0.841 / 0,359$ & 53 & 4 & $0.202 / 0.653$ & 45 & 12 & $0.562 / 0.454$ & 48 & 9 & $1.215 / 0.270$ \\
\hline $\begin{array}{l}\text { Dibioteca } \\
\text { virtual }\end{array}$ & No & 98 & 24 & $0,841 / 0,359$ & 111 & 11 & $0,202 / 0,053$ & 90 & 32 & $0,502 / 0,454$ & 94 & 28 & $1,215 / 0,2 / 0$ \\
\hline Uso de & $\mathrm{Si}$ & 100 & 18 & 163 & 108 & 10 & $0.004 / 0.949$ & 89 & 29 & $000 / 0998$ & 92 & 26 & $0393 / 0531$ \\
\hline datos & No & 47 & 14 & 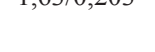 & 56 & 5 & ודיוסודסוס, & 46 & 15 & סוקים, & 50 & 11 & הכת, ודרנכ, \\
\hline
\end{tabular}




\begin{tabular}{|c|c|c|c|c|c|c|c|c|c|c|c|c|c|}
\hline \multirow{4}{*}{$\begin{array}{l}\text { Uso de las } \\
\text { TICS }\end{array}$} & Siempre & 30 & 6 & \multirow{4}{*}{$1,516 / 0,679$} & 34 & 2 & \multirow{4}{*}{$1,514 / 0,679$} & 32 & 4 & \multirow{4}{*}{$7,919 / 0,048$} & 28 & 8 & \multirow{4}{*}{$1,291 / 0,731$} \\
\hline & $\begin{array}{l}\text { Casi siem- } \\
\text { pre }\end{array}$ & 41 & 11 & & 46 & 6 & & 42 & 10 & & 44 & 8 & \\
\hline & $\begin{array}{l}\text { Algunas } \\
\text { veces }\end{array}$ & 71 & 15 & & 79 & 7 & & 58 & 28 & & 66 & 20 & \\
\hline & Nunca & 5 & 0 & & 5 & 0 & & 3 & 2 & & 4 & 1 & \\
\hline \multirow{4}{*}{$\begin{array}{l}\text { Métodos } \\
\text { usados por } \\
\text { los docentes } \\
\text { en clases } \\
\text { teóricas }\end{array}$} & $\begin{array}{l}\text { Clase ma- } \\
\text { gistral }\end{array}$ & 91 & 22 & \multirow{4}{*}{$0,792 / 0,851$} & 103 & 10 & \multirow{4}{*}{$1,724 / 0,632$} & 85 & 28 & \multirow{4}{*}{$3,168 / 0,366$} & 88 & 25 & \multirow{4}{*}{$0,871 / 0,832$} \\
\hline & $\begin{array}{l}\text { Análisis de } \\
\text { casos }\end{array}$ & 10 & 2 & & 11 & 1 & & 11 & 1 & & 9 & 3 & \\
\hline & $\begin{array}{l}\text { Seminarios } \\
\text { participa- } \\
\text { tivos }\end{array}$ & 33 & 5 & & 34 & 4 & & 29 & 9 & & 32 & 6 & \\
\hline & $\begin{array}{l}\text { Sociali- } \\
\text { zación de } \\
\text { guías de } \\
\text { trabajo } \\
\text { autónomo }\end{array}$ & 13 & 3 & & 16 & 0 & & 10 & 6 & & 13 & 3 & \\
\hline \multirow{5}{*}{$\begin{array}{l}\text { Métodos } \\
\text { que motivan } \\
\text { más el } \\
\text { aprendizaje }\end{array}$} & $\begin{array}{l}\text { Clase ma- } \\
\text { gistral }\end{array}$ & 25 & 3 & \multirow{5}{*}{$6,686 / 0,153$} & 26 & 2 & \multirow{5}{*}{$3,975 / 0,409$} & 23 & 5 & \multirow{5}{*}{$14,484 / 0,006$} & 27 & 1 & \multirow{5}{*}{$8,297 / 0,081$} \\
\hline & $\begin{array}{l}\text { Análisis de } \\
\text { casos }\end{array}$ & 52 & 10 & & 58 & 4 & & 43 & 19 & & 49 & 13 & \\
\hline & $\begin{array}{l}\text { Seminarios } \\
\text { participa- } \\
\text { tivos }\end{array}$ & 42 & 15 & & 49 & 8 & & 51 & 6 & & 44 & 13 & \\
\hline & $\begin{array}{l}\text { Sociali- } \\
\text { zación de } \\
\text { guías de } \\
\text { trabajo } \\
\text { autónomo }\end{array}$ & 24 & 2 & & 25 & 1 & & 15 & 11 & & 17 & 9 & \\
\hline & Otros & 4 & 2 & & 6 & 0 & & 3 & 3 & & 5 & 1 & \\
\hline
\end{tabular}

E-ISSN 2322-7028

Vol. 16 No. 2

May - Ago 2019

Cúcuta, Colombia

Fuente: Base de datos de las autoras

\section{Discusión}

En esta investigación se obtuvieron los resultados a partir de la aplicación del cuestionario CHAEA de Honey Alonso de estilos de aprendizaje, que mide los estilos activo, reflexivo, teórico y pragmático. En ese sentido, los hallazgos indican que dentro de las variables sociodemográficas la edad no fue considerada como un factor particular, relacionada con los estilos de aprendizaje; la edad mínima de los encuestados fue de 17 años y la edad máxima fue de 43 años. Estos datos comparados con estudios similares evidencian que el género y la edad no influyen en el estilo de aprendizaje (11), caso contrario con lo descrito por Villalba (12), quien demostró que existían preferencias importantes por el estilo reflexivo en los estudiantes menores de 21 años. Así mismo, Alducin y Vázquez (13), describen que existe menos puntuación en los estilos activo y reflexivo al aumentar la edad, sin tener influencia en los estilos teórico y pragmático.

De igual manera, en el contexto colombiano, Yancen et al.(6), refieren que los estudiantes de enfermería con respecto a las características sociodemográficas relacionadas con la edad oscilan entre 18 a 21 años y el sexo predominante es el femenino, lo cual, puede estar en concordancia con los aspectos históricos y culturales que han permeado el desarrollo histórico de la profesión a través del tiempo (14) y que aún persisten en la actualidad.

En cuanto a la correlación entre el nivel educativo de los padres o tutores otros estudios similares no han hallado relación significativa entre estas variables y las puntuaciones en los indicadores de rendimiento académico o en los resultados referidos a los estilos de aprendizaje (15).

Respecto, a los resultados relacionados con las motivaciones de los estudiantes encuestados para estudiar enfermería, predomina la motivación que tiene el estudiante para elegir la carrera como el deseo de ser siempre enfermero con un $66 \%$ de la población; al respecto, Talbot et al.(16), puntualizan que aunque las actitudes son aspectos de la vida individual, tienen una gran influencia en el desempeño y el com- 
ISSN-PRINT

1794-9831

E-ISSN 2322-7028

Vol. 16 No. 2

May - Ago 2019

Cúcuta, Colombia portamiento de los estudiantes del área de la salud, influyendo en el comportamiento de los integrantes de un grupo.

Además, el factor motivacional cobra doble sentido, en primer lugar, porque el motor interno promueve en el estudiante el impulso de elegir la enfermería como el camino para desarrollar su potencial humano; en segundo lugar, por la motivación que se promueve desde el proceso enseñanza aprendizaje y que surge de la experiencia vivida por parte del estudiante en la interacción constante con el nuevo conocimiento iniciado en las aulas.

Por otra parte, al indagar sobre los recursos educativos utilizados por los estudiantes para el aprendizaje, se evidenció que el $62,1 \%$ de la población encuestada asiste a la biblioteca para realizar búsqueda de información, aunque el 31,8\% utiliza libros de la biblioteca virtual, sin embargo llama la atención que solo el $48 \%$ de los participantes utiliza las TICS algunas veces. No obstante, se debe precisar que en el contexto global actual donde surge un nueva realidad social caracterizada por cambios dinámicos y avances acelerados en cuanto a ciencia, tecnología e información (17), exige que los profesionales de enfermería en formación adquieran competencias que no sólo estén centradas en las habilidades científico-técnicas, sino en la formación de ciudadanía y en el manejo de las tecnologías para el acceso rápido a la información; y de igual manera que les permita no solo acceder a ella sino transformarla y convertirla en conocimiento a favor del fortalecimiento del desempeño de la enfermera en su contexto.

Y aunque la investigación objeto del presente análisis no encontró correlación entre los factores sociodemográficos y los recursos educativos con los estilos de aprendizaje de los estudiantes, es necesario enfatizar sobre la importancia que tienen los métodos de enseñanza y los recursos que median y pueden favorecer el uso de un estilo de aprendizaje en ellos, más aún en la Educación Superior caracterizada por la diversidad de estudiantes, con clases más numerosas y menos homogéneas (18).

De igual manera, el conocimiento por parte de los docentes universitarios acerca de los estilos de aprendizaje puede determinar en un momento dado el uso de los recursos y métodos de enseñanza; por tanto, su importancia radica en que estos pueden promover en el estudiante la capacidad de aprender y el profesor debe saber cómo hacerlo (19).

En este sentido, al precisar el método utilizado por los docentes para la enseñanza, los resultados muestran que el método que predomina es la clase magistral, lo cual, se contradice con lo aludido por los encuestados, quienes señalan que los métodos didácticos que más facilitan el aprendizaje es el análisis de casos con un $62 \%$.

No obstante está situación contrasta con los hallazgos relacionados con la didáctica empleada por parte de los docentes en la enseñanza, predominando la clase magistral, frente a un porcentaje poco representativo sobre el uso de metodologías didácticas que propendan por desarrollar en el estudiante el análisis crítico enfocado a la toma de decisiones en enfermería. Este resultado se contradice con lo referido por la población encuestada, que describe en un $62 \%$ que el análisis de casos como método didáctico es el que más los anima a aprender. No obstante, esto concuerda con hallazgos descritos en estudios similares como el de Vizeshfar y Torabizadeh (20), en donde se expone que el $57.5 \%$ de los estudiantes de enfermería encuestados en su estudio, prefirió que sus profesores utilizaran métodos de participación y otros métodos que los involucrará y les permitiera ir más allá de los saberes aprendidos por ellos mismos.

Por su parte, Medina et al. (21) señalan que los estudiantes de enfermería durante su formación requieren del desarrollo de habilidades cognitivas orientadas a la observación, el análisis, la reflexión y la inferencia que les permita el desarrollo de un pensamiento crítico como base fundamental para el juicio clínico y para la toma de decisiones, ante una situación que demande su participación en bien de un paciente hospitalizado o de un colectivo.

En ese sentido, Benner (22) expresa que el pensamiento crítico es la base fundamental para la formación del conocimiento tácito de enfermería, es aquel conocimiento que le es propio a la enfermera, que aunque solo se genera en contacto con el paciente y la familia en el contexto clínico, también está cimentado sobre el desarrollo de habilidades cognitivas que lo favorezcan y que solo se propician bajo la realización de ejercicios prácticos desde el aula, como el análisis de casos clínicos que propendan por la toma de decisiones clínicas propias del profesional de enfermería en formación (23). 
Además, la calidad del profesor en las aulas requiere mucho más que el dominio de los contenidos de su área de especialización; su quehacer docente demanda de otros saberes pedagógicos, curriculares, contextuales, proposicionales y prácticos (21); así como la habilidad de manipularlos, transformarlos y conectarlos para dar una respuesta adecuada a cada situación de aula.

De ahí, que se haga hincapié sobre la importancia que tiene para los docentes el fomentar un buen aprendizaje en los estudiantes de enfermería de las diversas prácticas que se realizan desde el aula, lo cual, se revierte en un impacto positivo en el cuidado directo del paciente durante las prácticas académicas, según lo refieren Stirlin y Alquarini (24). En ese orden de ideas, la teoría propone un método para describir cómo lo estudiantes resuelven problemas y aplican el nuevo conocimiento desde su experiencia personal dentro del ambiente de aprendizaje (25).

En contraste con los hallazgos relacionados con los métodos de enseñanza, Stirlin y Alquarini (24) afirman que el desarrollo del conocimiento sobre los diferentes estilos de aprendizaje ayudarán a las facultades de enfermería a desarrollar los currículos, a adoptar métodos de enseñanza que los estudiantes disfruten y que al mismo tiempo generen un impacto en el ambiente de aprendizaje; factores didácticos semejantes a lo aportado por García et al.(26), quienes advierten sobre la importancia del conocimiento por parte de los docentes en los estilos de aprendizaje para la planeación de la enseñanza, basados en estrategias que no sólo privilegien el predominio de actitudes y dinámicas de la enseñanza tradicional.

Cabe señalar, que aunque en la presente investigación no se realizó un análisis comparativo entre las didácticas docentes y los estilos de aprendizaje, varios autores advierten que la incompatibilidad entre los estilos de enseñanza del docente y los estilos de aprendizaje del alumno son una barrera importante para el aprendizaje, por lo que es necesario que los educadores empleen una variedad de métodos para presentar su material y propiciar un aprendizaje significativo durante la formación (20).

Así mismo, se debe estimular en los estudiantes de enfermería durante la formación, la búsqueda de información que propicie en ellos competencias relacionadas con la autoformación, la investigación y la resolución de problemas. Desde esta perspectiva, Hernández et al. (27), afirman que los estudiantes en la búsqueda de información requerida para estudiar por medio del internet, utilizan un estilo de aprendizaje vinculado al afrontamiento cognitivo relacionado con la forma como el alumno interpreta que obtendrá éxito. Del mismo modo, el fortalecimiento en el uso del método de análisis de caso, favorece en los estudiantes de enfermería el desarrollo de la capacidad de pensamiento crítico (28), necesario para su ejercicio profesional en contexto; además, los casos de enfermería de alta calidad contribuyen a conectar de forma eficaz el conocimiento teórico con la práctica clínica (28).

De otro lado, algunos autores sustentan que conocer los estilos de aprendizaje de los estudiantes ayuda a los docentes no solo a planificar tareas adecuadas a sus preferencias, sino que también les sirve para promover su flexibilidad cognitiva, al proporcionarles tareas en las que tengan que emplear procedimientos diferentes a los habituales, que generen en el estudiante un nivel de comprensión sobre otras formas de resolver con éxito una tarea (27).

Al analizar los hallazgos relacionados con los estilos de aprendizaje se encontró que los estilos de aprendizaje que predominaron fueron el reflexivo con $15 \%$ y el teórico con el $14 \%$, siendo los que más prevalieron, seguidos por el pragmático con un 12,6\%. Estos resultados fueron similares a los descritos por Yancen et al. (6), quienes refieren en un estudio similar de corte cuantitativo, que el estilo de aprendizaje predominante fue el reflexivo, con un $36.7 \%$ de la población total, el teórico con $19.6 \%$ y el pragmático con 18.1 $\%$. Al respecto, Benner (22) hace explícito que en la formación del pensamiento clínico, los estudiantes son considerados en los inicios de su formación, enfermeros novicios, refiriéndose al conocimiento que es aprendido a través de conceptos, reglas y normas, como base para conocer los aspectos que hacen parte de la disciplina y del cuidado, conocimientos que solo se consolidan en la relación enfermera paciente en el contexto clínico; sin embargo, su fase de formación la inicia desde los primeros saberes que son enseñados en el aula.

Sin embargo, Isaza (3) al aplicar el instrumento CHAEA en estudiantes de enfermería, afirma que aunque los estilos de aprendizaje no se presentan de manera única, hubo un predominio por los estilos pragmático y teórico. Según la autora, estos estilos 
ISSN-PRINT

1794-9831

E-ISSN 2322-7028

Vol. 16 No. 2

May - Ago 2019

Cúcuta, Colombia se encuentran asociados a los modelos tradicionales, presentes en las experiencias escolares previas de los estudiantes.

Según los resultados, un hallazgo que llama la atención se relaciona con la integración de varios estilos de aprendizaje por parte de los estudiantes encuestados en esta investigación; se puede observar, que en la medida que avanzan en los semestres, pasan de un estilo teórico y reflexivo con un $89 \%$ y un $83,3 \%$ en el primer semestre, a un estilo de aprendizaje activo con $96,2 \%$ y ponderando el reflexivo y el pragmático con un $88,2 \%$ en el tercer semestre. Estos hallazgos son similares a los encontrados por Van (29) en Amsterdam, quien afirma que los estudiantes son capaces de desarrollar un aprendizaje que integre diferentes estilos de aprendizaje, lo cual se hace más evidente en la medida en que los estudiantes avanzan hacia los semestres superiores durante la formación universitaria.

Por su parte, Gonzalez et al. (1), mediante la aplicación del instrumento CHAEA, reportan que la mayor parte de los estudiantes tienen tres o más estilos de aprendizaje, y aunque al relacionarlo con el desempeño académico no se encontró significancia estadística, las autoras resaltan sobre la importancia que tiene el conocimiento de los estilos de aprendizaje para facilitar el desarrollo de técnicas y estrategias de enseñanza más efectivas, que propicien un clima más acogedor y promueva la participación de los estudiantes (1). También se encontró similitud con hallazgos de otra investigación realizada en estudiantes de enfermería en Nueva Zelanda, donde se comprobó que los estudiantes universitarios utilizan diversos métodos para llevar a cabo el aprendizaje, entre ellos, la inclusión de la tecnología en línea y táctil, como los medios más preferidos (30). De la misma manera, se comprobó que los docentes de enfermería, deben trascender los modelos tradicionales educativos y conocer los estilos de aprendizaje para adaptar la didáctica, incluyendo recursos innovadores para hacer del proceso enseñanza aprendizaje una experiencia significativa durante el proceso formativo.

Es interesante analizar la forma como los estudiantes de los semestres avanzados logran combinar los tres estilos de aprendizaje ponderados: el activo, reflexivo y teórico; sin embargo, se encontraron algunas diferencias particulares en el quinto y noveno semestre donde, según el Baremo, por lo menos un estilo, se puntúa como muy alto, evento que no aparece en los otros semestres. Si se tiene en cuenta el contexto académico del programa de formación objeto del análisis, en el quinto semestre los estudiantes entran a un nuevo ciclo de prácticas académicas, donde se sumergen más en el cuidado directo de las personas, respecto a los semestres anteriores donde estaban más enfocados hacia el conocimiento teórico y a la ejecución de los procedimientos técnicos inherentes a la disciplina de la enfermería. Así mismo, aunque en los semestres 6,7 y 8 , las puntuaciones fueron muy estables, no obstante se notó disminución respecto a los cinco primeros semestres de formación en la aplicación de los diferentes estilos, situación que cambia en el noveno semestre que se vuelve a presentar una integración de los estilos activo, reflexivo y teórico.

De modo similar, en el noveno semestre también ocurren cambios significativos en el proceso académico, debido sobre todo, a que los estudiantes pasan a ser autónomos en la práctica, tienen nuevos roles y se centran en la gestión integral de los servicios de enfermería; es decir, que esta tendencia está orientada a potencializar los cuatro estilos, por tanto está acorde con la situación a la que se someten los estudiantes, ya que se enfrentan no solo a las situaciones clínicas o comunitarias con las personas sujetos de cuidado, sino también a liderar servicios clínicos o de salud pública. Los aspectos indicados anteriormente están en concordancia, con lo expuesto por Benner (22) quien expone que el estudiante novicio empieza a trascender en su nivel de pensamiento enfermero a niveles más avanzados, caracterizados por pensamiento objetivo, análisis de aspectos que hacen parte de una situación de cuidado, dependiendo de la interacción que tiene con el paciente y la familia. Es de resaltar, que si bien la autora rescata la importancia del contexto clínico en la formación del pensamiento enfermero, este proceso no sólo es propio de la práctica clínica, sino que también se inicia desde que el estudiante aprende los saberes teóricos del cuidado de enfermería en el aula (31).

En otras palabras, este estudio permite demostrar que los estudiantes no modifican sus preferencias de estilos, pues los continúan usando a través de su formación, con los cambios particulares del contexto académico a los que se enfrentan al empezar a integrar los saberes teóricos durante las prácticas desde el quinto semestre; por tanto, requieren potencializar sus estilos porque corresponderán a los requerimientos del entorno en los diferentes ambientes de aprendizaje donde llevan a cabo las prácticas de cuidado. En este sentido, Stirling (32) expone en su estudio que se debe analizar la importancia sobre el conocimiento de los estilos de 
aprendizaje de los estudiantes de enfermería durante la formación; igualmente, hay que tener en cuenta que los docentes reconocen que están preparando a sus estudiantes para ser aprendices de por vida. También hay que precisar, que las enfermeras deben aprender sobre sus sujetos de cuidado en la práctica, mediante una variedad de formas diferentes, que implican desde leer gráficos, observar y evaluar, escuchar informes orales y a los pacientes, es decir, desarrollar habilidades clínicas (22). Esta es la forma en que las enfermeras aprenden en los años universitarios que las prepararán para convertirse en aprendices de por vida (32).

En consecuencia, nuevos retos enfrentan los docentes universitarios en la formación de enfermeras en la actualidad, que van más allá del conocimiento de una asignatura; por tanto, es fundamental que el docente propicie el aprendizaje de saberes por medio del uso de didácticas que lo posibiliten (33).

Sobre la base de las ideas expuestas, hay que dirigir la mirada hacia las demandas que surgen del contexto, de manera que permitan la integración de competencias que tienen implícita la actuación profesional en la esfera social, económica, el desarrollo tecnológico, los cambios demográficos, epidemiológicos y el medioambiente (34).

Así mismo, Gonzáles et al. (35), demostraron que el uso de técnicas de aprendizaje auto dirigido hicieron que los alumnos participaran en su formación empleando de diferentes métodos, como la realización de esquemas y mapas conceptuales para mejorar la comprensión de los contenidos, la implementación de la planificación para la elaboración de material propio como videos y folletos o de actividades de trabajo de campo; actividades que favorecen la autonomía y el trabajo en equipo, competencias que son inherentes al ejercicio de la profesión en cualquier contexto.

En resumen, es preciso rescatar la importancia del uso de nuevas metodologías de enseñanza, así como la implementación de recursos didácticos innovadores y la flexibilidad en los estilos, para llevar a cabo la construcción del conocimiento por parte de los docentes encargados de la enseñanza de los saberes teóricos. Actividades que se deben realizar con el objetivo de propiciar el desarrollo y fortalecimiento de los diversos estilos de aprendizaje por parte de los estudiante en cada semestre; siempre teniendo en cuenta que las necesidades de los estudiantes son variadas y están determinadas no solo por sus necesidades de aprendizaje, sino por las competencias planteadas en cada currículo, como lo refiere Gimeno (36). Es por eso, que el uso de métodos de enseñanza homogéneos no representan la mejor opción para la enseñanza en el aula, como lo afirma Flórez (37).

Conclusiones
Los resultados de la investigación no evidenciaron
asociación estadística entre los estilos de apren-
dizaje y las variables sociodemográficas, perso-
nales y de recursos educativos; no obstante, aún
persiste un predominio del sexo femenino en la po-
blación que hace parte de la formación de la enfer-
mería como profesión, lo cual, concuerda con los
hallazgos referidos en otros estudios y puede estar
influenciado por rasgos culturales que han carac-
terizado el desarrollo histórico de la disciplina.
Los métodos didácticos utilizados por los docen-
tes, según ésta investigación, emplean en un alto
porcentaje la clase magistral, método que en los
primeros semestres de formación del pensamien-
to en la enfermería puede ser consecuente con el
uso de modelos tradicionales de educación. Sin
embargo, existen bases conceptuales en las asig-
naturas relacionadas con las ciencias básicas que
pueden tener un predominio teórico, pero que al
persistir en ellas, poco contribuyen a la formación
de habilidades del pensamiento superior como la
observación, el análisis, la inferencia, para que
contribuyan al desarrollo de competencias espe-
cíficas de la enfermera, encaminadas a la toma de
decisiones en contexto, para favorecer las perso-
nas y colectivos sujetos de cuidado.
Los estudiantes del programa de enfermería ob-
jeto de este estudio aplican los diferentes esti-
los de aprendizaje en los diversos semestres; sin
embargo, existe una tendencia a recurrir al estilo
de aprendizaje reflexivo y teórico, lo que con-
stituyen nuevos retos para los docentes, que van
desde un conocimiento no sólo del área especí-
fica objeto de enseñanza, hasta el fortalecimiento
de los diversos métodos, estrategias y recursos
didácticos, para propiciar en los estudiantes apre-
ndizajes significativos desde el aula.
Conflicto de Intereses

Conclusiones
Los resultados de la investigación no evidenciaron
asociación estadística entre los estilos de apren-
dizaje y las variables sociodemográficas, perso-
nales y de recursos educativos; no obstante, aún
persiste un predominio del sexo femenino en la po-
blación que hace parte de la formación de la enfer-
mería como profesión, lo cual, concuerda con los
hallazgos referidos en otros estudios y puede estar
influenciado por rasgos culturales que han carac-
terizado el desarrollo histórico de la disciplina.
Los métodos didácticos utilizados por los docen-
tes, según ésta investigación, emplean en un alto
porcentaje la clase magistral, método que en los
primeros semestres de formación del pensamien-
to en la enfermería puede ser consecuente con el
uso de modelos tradicionales de educación. Sin
embargo, existen bases conceptuales en las asig-
naturas relacionadas con las ciencias básicas que
pueden tener un predominio teórico, pero que al
persistir en ellas, poco contribuyen a la formación
de habilidades del pensamiento superior como la
observación, el análisis, la inferencia, para que
contribuyan al desarrollo de competencias espe-
cíficas de la enfermera, encaminadas a la toma de
decisiones en contexto, para favorecer las perso-
nas y colectivos sujetos de cuidado.
Los estudiantes del programa de enfermería ob-
jeto de este estudio aplican los diferentes esti-
los de aprendizaje en los diversos semestres; sin
embargo, existe una tendencia a recurrir al estilo
de aprendizaje reflexivo y teórico, lo que con-
stituyen nuevos retos para los docentes, que van
desde un conocimiento no sólo del área especí-
fica objeto de enseñanza, hasta el fortalecimiento
de los diversos métodos, estrategias y recursos
divas estudiantes apre-
autoses desde el aula.
-

Conclusiones
Los resultados de la investigación no evidenciaron
asociación estadística entre los estilos de apren-
dizaje y las variables sociodemográficas, perso-
nales y de recursos educativos; no obstante, aún
persiste un predominio del sexo femenino en la po-
blación que hace parte de la formación de la enfer-
mería como profesión, lo cual, concuerda con los
hallazgos referidos en otros estudios y puede estar
influenciado por rasgos culturales que han carac-
terizado el desarrollo histórico de la disciplina.
Los métodos didácticos utilizados por los docen-
tes, según ésta investigación, emplean en un alto
porcentaje la clase magistral, método que en los
primeros semestres de formación del pensamien-
to en la enfermería puede ser consecuente con el
uso de modelos tradicionales de educación. Sin
embargo, existen bases conceptuales en las asig-
naturas relacionadas con las ciencias básicas que
pueden tener un predominio teórico, pero que al
persistir en ellas, poco contribuyen a la formación
de habilidades del pensamiento superior como la
observación, el análisis, la inferencia, para que
contribuyan al desarrollo de competencias espe-
cíficas de la enfermera, encaminadas a la toma de
decisiones en contexto, para favorecer las perso-
nas y colectivos sujetos de cuidado.
Los estudiantes del programa de enfermería ob-
jeto de este estudio aplican los diferentes esti-
los de aprendizaje en los diversos semestres; sin
embargo, existe una tendencia a recurrir al estilo
de aprendizaje reflexivo y teórico, lo que con-
stituyen nuevos retos para los docentes, que van
desde un conocimiento no sólo del área especí-
fica objeto de enseñanza, hasta el fortalecimiento
de los diversos métodos, estrategias y recursos
didácticos, para propiciar en los estudiantes apre-
ndizajes significativos desde el aula.
-

Conclusiones
Los resultados de la investigación no evidenciaron
asociación estadística entre los estilos de apren-
dizaje y las variables sociodemográficas, perso-
nales y de recursos educativos; no obstante, aún
persiste un predominio del sexo femenino en la po-
blación que hace parte de la formación de la enfer-
mería como profesión, lo cual, concuerda con los
hallazgos referidos en otros estudios y puede estar
influenciado por rasgos culturales que han carac-
terizado el desarrollo histórico de la disciplina.
Los métodos didácticos utilizados por los docen-
tes, según ésta investigación, emplean en un alto
porcentaje la clase magistral, método que en los
primeros semestres de formación del pensamien-
to en la enfermería puede ser consecuente con el
uso de modelos tradicionales de educación. Sin
embargo, existen bases conceptuales en las asig-
naturas relacionadas con las ciencias básicas que
pueden tener un predominio teórico, pero que al
persistir en ellas, poco contribuyen a la formación
de habilidades del pensamiento superior como la
observación, el análisis, la inferencia, para que
contribuyan al desarrollo de competencias espe-
cíficas de la enfermera, encaminadas a la toma de
decisiones en contexto, para favorecer las perso-
nas y colectivos sujetos de cuidado.
Los estudiantes del programa de enfermería ob-
jeto de este estudio aplican los diferentes esti-
los de aprendizaje en los diversos semestres; sin
embargo, existe una tendencia a recurrir al estilo
de aprendizaje reflexivo y teórico, lo que con-
stituyen nuevos retos para los docentes, que van
desde un conocimiento no sólo del área especí-
fica objeto de enseñanza, hasta el fortalecimiento
de los diversos métodos, estrategias y recursos
didácticos, para propiciar en los estudiantes apre-
ndizajes significativos desde el aula.
-

Conclusiones
Los resultados de la investigación no evidenciaron
asociación estadística entre los estilos de apren-
dizaje y las variables sociodemográficas, perso-
nales y de recursos educativos; no obstante, aún
persiste un predominio del sexo femenino en la po-
blación que hace parte de la formación de la enfer-
mería como profesión, lo cual, concuerda con los
hallazgos referidos en otros estudios y puede estar
influenciado por rasgos culturales que han carac-
terizado el desarrollo histórico de la disciplina.
Los métodos didácticos utilizados por los docen-
tes, según ésta investigación, emplean en un alto
porcentaje la clase magistral, método que en los
primeros semestres de formación del pensamien-
to en la enfermería puede ser consecuente con el
uso de modelos tradicionales de educación. Sin
embargo, existen bases conceptuales en las asig-
naturas relacionadas con las ciencias básicas que
pueden tener un predominio teórico, pero que al
persistir en ellas, poco contribuyen a la formación
de habilidades del pensamiento superior como la
observación, el análisis, la inferencia, para que
contribuyan al desarrollo de competencias espe-
cíficas de la enfermera, encaminadas a la toma de
decisiones en contexto, para favorecer las perso-
nas y colectivos sujetos de cuidado.
Los estudiantes del programa de enfermería ob-
jeto de este estudio aplican los diferentes esti-
los de aprendizaje en los diversos semestres; sin
embargo, existe una tendencia a recurrir al estilo
de aprendizaje reflexivo y teórico, lo que con-
stituyen nuevos retos para los docentes, que van
desde un conocimiento no sólo del área especí-
fica objeto de enseñanza, hasta el fortalecimiento
de los diversos métodos, estrategias y recursos
didácticos, para propiciar en los estudiantes apre-
ndizajes significativos desde el aula.
-

Conclusiones
Los resultados de la investigación no evidenciaron
asociación estadística entre los estilos de apren-
dizaje y las variables sociodemográficas, perso-
nales y de recursos educativos; no obstante, aún
persiste un predominio del sexo femenino en la po-
blación que hace parte de la formación de la enfer-
mería como profesión, lo cual, concuerda con los
hallazgos referidos en otros estudios y puede estar
influenciado por rasgos culturales que han carac-
terizado el desarrollo histórico de la disciplina.
Los métodos didácticos utilizados por los docen-
tes, según ésta investigación, emplean en un alto
porcentaje la clase magistral, método que en los
primeros semestres de formación del pensamien-
to en la enfermería puede ser consecuente con el
uso de modelos tradicionales de educación. Sin
embargo, existen bases conceptuales en las asig-
naturas relacionadas con las ciencias básicas que
pueden tener un predominio teórico, pero que al
persistir en ellas, poco contribuyen a la formación
de habilidades del pensamiento superior como la
observación, el análisis, la inferencia, para que
contribuyan al desarrollo de competencias espe-
cíficas de la enfermera, encaminadas a la toma de
decisiones en contexto, para favorecer las perso-
nas y colectivos sujetos de cuidado.
Los estudiantes del programa de enfermería ob-
jeto de este estudio aplican los diferentes esti-
los de aprendizaje en los diversos semestres; sin
embargo, existe una tendencia a recurrir al estilo
de aprendizaje reflexivo y teórico, lo que con-
stituyen nuevos retos para los docentes, que van
desde un conocimiento no sólo del área especí-
fica objeto de enseñanza, hasta el fortalecimiento
de los diversos métodos, estrategias y recursos
didácticos, para propiciar en los estudiantes apre-
ndizajes significativos desde el aula.
-

Conclusiones
Los resultados de la investigación no evidenciaron
asociación estadística entre los estilos de apren-
dizaje y las variables sociodemográficas, perso-
nales y de recursos educativos; no obstante, aún
persiste un predominio del sexo femenino en la po-
blación que hace parte de la formación de la enfer-
mería como profesión, lo cual, concuerda con los
hallazgos referidos en otros estudios y puede estar
influenciado por rasgos culturales que han carac-
terizado el desarrollo histórico de la disciplina.
Los métodos didácticos utilizados por los docen-
tes, según ésta investigación, emplean en un alto
porcentaje la clase magistral, método que en los
primeros semestres de formación del pensamien-
to en la enfermería puede ser consecuente con el
uso de modelos tradicionales de educación. Sin
embargo, existen bases conceptuales en las asig-
naturas relacionadas con las ciencias básicas que
pueden tener un predominio teórico, pero que al
persistir en ellas, poco contribuyen a la formación
de habilidades del pensamiento superior como la
observación, el análisis, la inferencia, para que
contribuyan al desarrollo de competencias espe-
cíficas de la enfermera, encaminadas a la toma de
decisiones en contexto, para favorecer las perso-
nas y colectivos sujetos de cuidado.
Los estudiantes del programa de enfermería ob-
jeto de este estudio aplican los diferentes esti-
los de aprendizaje en los diversos semestres; sin
embargo, existe una tendencia a recurrir al estilo
de aprendizaje reflexivo y teórico, lo que con-
stituyen nuevos retos para los docentes, que van
desde un conocimiento no sólo del área especí-
fica objeto de enseñanza, hasta el fortalecimiento
de los diversos métodos, estrategias y recursos
didácticos, para propiciar en los estudiantes apre-
ndizajes significativos desde el aula.
-

Conclusiones
Los resultados de la investigación no evidenciaron
asociación estadística entre los estilos de apren-
dizaje y las variables sociodemográficas, perso-
nales y de recursos educativos; no obstante, aún
persiste un predominio del sexo femenino en la po-
blación que hace parte de la formación de la enfer-
mería como profesión, lo cual, concuerda con los
hallazgos referidos en otros estudios y puede estar
influenciado por rasgos culturales que han carac-
terizado el desarrollo histórico de la disciplina.
Los métodos didácticos utilizados por los docen-
tes, según ésta investigación, emplean en un alto
porcentaje la clase magistral, método que en los
primeros semestres de formación del pensamien-
to en la enfermería puede ser consecuente con el
uso de modelos tradicionales de educación. Sin
embargo, existen bases conceptuales en las asig-
naturas relacionadas con las ciencias básicas que
pueden tener un predominio teórico, pero que al
persistir en ellas, poco contribuyen a la formación
de habilidades del pensamiento superior como la
observación, el análisis, la inferencia, para que
contribuyan al desarrollo de competencias espe-
cíficas de la enfermera, encaminadas a la toma de
decisiones en contexto, para favorecer las perso-
nas y colectivos sujetos de cuidado.
Los estudiantes del programa de enfermería ob-
jeto de este estudio aplican los diferentes esti-
los de aprendizaje en los diversos semestres; sin
embargo, existe una tendencia a recurrir al estilo
de aprendizaje reflexivo y teórico, lo que con-
stituyen nuevos retos para los docentes, que van
desde un conocimiento no sólo del área especí-
fica objeto de enseñanza, hasta el fortalecimiento
de los diversos métodos, estrategias y recursos
didácticos, para propiciar en los estudiantes apre-
ndizajes significativos desde el aula.
-

Conclusiones
Los resultados de la investigación no evidenciaron
asociación estadística entre los estilos de apren-
dizaje y las variables sociodemográficas, perso-
nales y de recursos educativos; no obstante, aún
persiste un predominio del sexo femenino en la po-
blación que hace parte de la formación de la enfer-
mería como profesión, lo cual, concuerda con los
hallazgos referidos en otros estudios y puede estar
influenciado por rasgos culturales que han carac-
terizado el desarrollo histórico de la disciplina.
Los métodos didácticos utilizados por los docen-
tes, según ésta investigación, emplean en un alto
porcentaje la clase magistral, método que en los
primeros semestres de formación del pensamien-
to en la enfermería puede ser consecuente con el
uso de modelos tradicionales de educación. Sin
embargo, existen bases conceptuales en las asig-
naturas relacionadas con las ciencias básicas que
pueden tener un predominio teórico, pero que al
persistir en ellas, poco contribuyen a la formación
de habilidades del pensamiento superior como la
observación, el análisis, la inferencia, para que
contribuyan al desarrollo de competencias espe-
cíficas de la enfermera, encaminadas a la toma de
decisiones en contexto, para favorecer las perso-
nas y colectivos sujetos de cuidado.
Los estudiantes del programa de enfermería ob-
jeto de este estudio aplican los diferentes esti-
los de aprendizaje en los diversos semestres; sin
embargo, existe una tendencia a recurrir al estilo
de aprendizaje reflexivo y teórico, lo que con-
stituyen nuevos retos para los docentes, que van
desde un conocimiento no sólo del área especí-
fica objeto de enseñanza, hasta el fortalecimiento
de los diversos métodos, estrategias y recursos
didácticos, para propiciar en los estudiantes apre-
ndizajes significativos desde el aula.
-

Conclusiones
Los resultados de la investigación no evidenciaron
asociación estadística entre los estilos de apren-
dizaje y las variables sociodemográficas, perso-
nales y de recursos educativos; no obstante, aún
persiste un predominio del sexo femenino en la po-
blación que hace parte de la formación de la enfer-
mería como profesión, lo cual, concuerda con los
hallazgos referidos en otros estudios y puede estar
influenciado por rasgos culturales que han carac-
terizado el desarrollo histórico de la disciplina.
Los métodos didácticos utilizados por los docen-
tes, según ésta investigación, emplean en un alto
porcentaje la clase magistral, método que en los
primeros semestres de formación del pensamien-
to en la enfermería puede ser consecuente con el
uso de modelos tradicionales de educación. Sin
embargo, existen bases conceptuales en las asig-
naturas relacionadas con las ciencias básicas que
pueden tener un predominio teórico, pero que al
persistir en ellas, poco contribuyen a la formación
de habilidades del pensamiento superior como la
observación, el análisis, la inferencia, para que
contribuyan al desarrollo de competencias espe-
cíficas de la enfermera, encaminadas a la toma de
decisiones en contexto, para favorecer las perso-
nas y colectivos sujetos de cuidado.
Los estudiantes del programa de enfermería ob-
jeto de este estudio aplican los diferentes esti-
los de aprendizaje en los diversos semestres; sin
embargo, existe una tendencia a recurrir al estilo
de aprendizaje reflexivo y teórico, lo que con-
stituyen nuevos retos para los docentes, que van
desde un conocimiento no sólo del área especí-
fica objeto de enseñanza, hasta el fortalecimiento
de los diversos métodos, estrategias y recursos
didácticos, para propiciar en los estudiantes apre-
ndizajes significativos desde el aula.
-

Conclusiones
Los resultados de la investigación no evidenciaron
asociación estadística entre los estilos de apren-
dizaje y las variables sociodemográficas, perso-
nales y de recursos educativos; no obstante, aún
persiste un predominio del sexo femenino en la po-
blación que hace parte de la formación de la enfer-
mería como profesión, lo cual, concuerda con los
hallazgos referidos en otros estudios y puede estar
influenciado por rasgos culturales que han carac-
terizado el desarrollo histórico de la disciplina.
Los métodos didácticos utilizados por los docen-
tes, según ésta investigación, emplean en un alto
porcentaje la clase magistral, método que en los
primeros semestres de formación del pensamien-
to en la enfermería puede ser consecuente con el
uso de modelos tradicionales de educación. Sin
embargo, existen bases conceptuales en las asig-
naturas relacionadas con las ciencias básicas que
pueden tener un predominio teórico, pero que al
persistir en ellas, poco contribuyen a la formación
de habilidades del pensamiento superior como la
observación, el análisis, la inferencia, para que
contribuyan al desarrollo de competencias espe-
cíficas de la enfermera, encaminadas a la toma de
decisiones en contexto, para favorecer las perso-
nas y colectivos sujetos de cuidado.
Los estudiantes del programa de enfermería ob-
jeto de este estudio aplican los diferentes esti-
los de aprendizaje en los diversos semestres; sin
embargo, existe una tendencia a recurrir al estilo
de aprendizaje reflexivo y teórico, lo que con-
stituyen nuevos retos para los docentes, que van
desde un conocimiento no sólo del área especí-
fica objeto de enseñanza, hasta el fortalecimiento
de los diversos métodos, estrategias y recursos
didácticos, para propiciar en los estudiantes apre-
ndizajes significativos desde el aula.
-

Conclusiones
Los resultados de la investigación no evidenciaron
asociación estadística entre los estilos de apren-
dizaje y las variables sociodemográficas, perso-
nales y de recursos educativos; no obstante, aún
persiste un predominio del sexo femenino en la po-
blación que hace parte de la formación de la enfer-
mería como profesión, lo cual, concuerda con los
hallazgos referidos en otros estudios y puede estar
influenciado por rasgos culturales que han carac-
terizado el desarrollo histórico de la disciplina.
Los métodos didácticos utilizados por los docen-
tes, según ésta investigación, emplean en un alto
porcentaje la clase magistral, método que en los
primeros semestres de formación del pensamien-
to en la enfermería puede ser consecuente con el
uso de modelos tradicionales de educación. Sin
embargo, existen bases conceptuales en las asig-
naturas relacionadas con las ciencias básicas que
pueden tener un predominio teórico, pero que al
persistir en ellas, poco contribuyen a la formación
de habilidades del pensamiento superior como la
observación, el análisis, la inferencia, para que
contribuyan al desarrollo de competencias espe-
cíficas de la enfermera, encaminadas a la toma de
decisiones en contexto, para favorecer las perso-
nas y colectivos sujetos de cuidado.
jeto de este estudio aplican los diferentes esti-
los de aprendizaje en los diversos semestres; sin
embargo, existe una tendencia a recurrir al estilo
de aprendizaje reflexivo y teórico, lo que con-
stituyen nuevos retos para los docentes, que van
desde un conocimiento no sólo del área especí-
fica objeto de enseñanza, hasta el fortalecimiento
de los diversos métodos, estrategias y recursos
dignificativiciar en los estudiantes apre-
-

Conclusiones
Los resultados de la investigación no evidenciaron
asociación estadística entre los estilos de apren-
dizaje y las variables sociodemográficas, perso-
nales y de recursos educativos; no obstante, aún
persiste un predominio del sexo femenino en la po-
blación que hace parte de la formación de la enfer-
mería como profesión, lo cual, concuerda con los
hallazgos referidos en otros estudios y puede estar
influenciado por rasgos culturales que han carac-
terizado el desarrollo histórico de la disciplina.
Los métodos didácticos utilizados por los docen-
tes, según ésta investigación, emplean en un alto
porcentaje la clase magistral, método que en los
primeros semestres de formación del pensamien-
to en la enfermería puede ser consecuente con el
uso de modelos tradicionales de educación. Sin
embargo, existen bases conceptuales en las asig-
naturas relacionadas con las ciencias básicas que
pueden tener un predominio teórico, pero que al
persistir en ellas, poco contribuyen a la formación
de habilidades del pensamiento superior como la
observación, el análisis, la inferencia, para que
contribuyan al desarrollo de competencias espe-
cíficas de la enfermera, encaminadas a la toma de
decisiones en contexto, para favorecer las perso-
nas y colectivos sujetos de cuidado.
Los estudiantes del programa de enfermería ob-
jeto de este estudio aplican los diferentes esti-
los de aprendizaje en los diversos semestres; sin
embargo, existe una tendencia a recurrir al estilo
de aprendizaje reflexivo y teórico, lo que con-
stituyen nuevos retos para los docentes, que van
desde un conocimiento no sólo del área especí-
fica objeto de enseñanza, hasta el fortalecimiento
de los diversos métodos, estrategias y recursos
didácticos, para propiciar en los estudiantes apre-
ndizajes significativos desde el aula.
-

Conclusiones
Los resultados de la investigación no evidenciaron
asociación estadística entre los estilos de apren-
dizaje y las variables sociodemográficas, perso-
nales y de recursos educativos; no obstante, aún
persiste un predominio del sexo femenino en la po-
blación que hace parte de la formación de la enfer-
mería como profesión, lo cual, concuerda con los
hallazgos referidos en otros estudios y puede estar
influenciado por rasgos culturales que han carac-
terizado el desarrollo histórico de la disciplina.
Los métodos didácticos utilizados por los docen-
tes, según ésta investigación, emplean en un alto
porcentaje la clase magistral, método que en los
primeros semestres de formación del pensamien-
to en la enfermería puede ser consecuente con el
uso de modelos tradicionales de educación. Sin
embargo, existen bases conceptuales en las asig-
naturas relacionadas con las ciencias básicas que
pueden tener un predominio teórico, pero que al
persistir en ellas, poco contribuyen a la formación
de habilidades del pensamiento superior como la
observación, el análisis, la inferencia, para que
contribuyan al desarrollo de competencias espe-
cíficas de la enfermera, encaminadas a la toma de
decisiones en contexto, para favorecer las perso-
nas y colectivos sujetos de cuidado.
Los estudiantes del programa de enfermería ob-
jeto de este estudio aplican los diferentes esti-
los de aprendizaje en los diversos semestres; sin
embargo, existe una tendencia a recurrir al estilo
de aprendizaje reflexivo y teórico, lo que con-
stituyen nuevos retos para los docentes, que van
desde un conocimiento no sólo del área especí-
fica objeto de enseñanza, hasta el fortalecimiento
de los diversos métodos, estrategias y recursos
didácticos, para propiciar en los estudiantes apre-
ndizajes significativos desde el aula.
-

Conclusiones
Los resultados de la investigación no evidenciaron
asociación estadística entre los estilos de apren-
dizaje y las variables sociodemográficas, perso-
nales y de recursos educativos; no obstante, aún
persiste un predominio del sexo femenino en la po-
blación que hace parte de la formación de la enfer-
mería como profesión, lo cual, concuerda con los
hallazgos referidos en otros estudios y puede estar
influenciado por rasgos culturales que han carac-
terizado el desarrollo histórico de la disciplina.
Los métodos didácticos utilizados por los docen-
tes, según ésta investigación, emplean en un alto
porcentaje la clase magistral, método que en los
primeros semestres de formación del pensamien-
to en la enfermería puede ser consecuente con el
uso de modelos tradicionales de educación. Sin
embargo, existen bases conceptuales en las asig-
naturas relacionadas con las ciencias básicas que
pueden tener un predominio teórico, pero que al
persistir en ellas, poco contribuyen a la formación
de habilidades del pensamiento superior como la
observación, el análisis, la inferencia, para que
contribuyan al desarrollo de competencias espe-
cíficas de la enfermera, encaminadas a la toma de
decisiones en contexto, para favorecer las perso-
nas y colectivos sujetos de cuidado.
Los estudiantes del programa de enfermería ob-
jeto de este estudio aplican los diferentes esti-
los de aprendizaje en los diversos semestres; sin
embargo, existe una tendencia a recurrir al estilo
de aprendizaje reflexivo y teórico, lo que con-
stituyen nuevos retos para los docentes, que van
desde un conocimiento no sólo del área especí-
fica objeto de enseñanza, hasta el fortalecimiento
de los diversos métodos, estrategias y recursos
didácticos, para propiciar en los estudiantes apre-
ndizajes significativos desde el aula.
-

Conclusiones
Los resultados de la investigación no evidenciaron
asociación estadística entre los estilos de apren-
dizaje y las variables sociodemográficas, perso-
nales y de recursos educativos; no obstante, aún
persiste un predominio del sexo femenino en la po-
blación que hace parte de la formación de la enfer-
mería como profesión, lo cual, concuerda con los
hallazgos referidos en otros estudios y puede estar
influenciado por rasgos culturales que han carac-
terizado el desarrollo histórico de la disciplina.
Los métodos didácticos utilizados por los docen-
tes, según ésta investigación, emplean en un alto
porcentaje la clase magistral, método que en los
primeros semestres de formación del pensamien-
to en la enfermería puede ser consecuente con el
uso de modelos tradicionales de educación. Sin
embargo, existen bases conceptuales en las asig-
naturas relacionadas con las ciencias básicas que
pueden tener un predominio teórico, pero que al
persistir en ellas, poco contribuyen a la formación
de habilidades del pensamiento superior como la
observación, el análisis, la inferencia, para que
contribuyan al desarrollo de competencias espe-
cíficas de la enfermera, encaminadas a la toma de
decisiones en contexto, para favorecer las perso-
nas y colectivos sujetos de cuidado.
Los estudiantes del programa de enfermería ob-
jeto de este estudio aplican los diferentes esti-
los de aprendizaje en los diversos semestres; sin
embargo, existe una tendencia a recurrir al estilo
de aprendizaje reflexivo y teórico, lo que con-
stituyen nuevos retos para los docentes, que van
desde un conocimiento no sólo del área especí-
fica objeto de enseñanza, hasta el fortalecimiento
de los diversos métodos, estrategias y recursos
didácticos, para propiciar en los estudiantes apre-
ndizajes significativos desde el aula.
-

Conclusiones
Los resultados de la investigación no evidenciaron
asociación estadística entre los estilos de apren-
dizaje y las variables sociodemográficas, perso-
nales y de recursos educativos; no obstante, aún
persiste un predominio del sexo femenino en la po-
blación que hace parte de la formación de la enfer-
mería como profesión, lo cual, concuerda con los
hallazgos referidos en otros estudios y puede estar
influenciado por rasgos culturales que han carac-
terizado el desarrollo histórico de la disciplina.
Los métodos didácticos utilizados por los docen-
tes, según ésta investigación, emplean en un alto
porcentaje la clase magistral, método que en los
primeros semestres de formación del pensamien-
to en la enfermería puede ser consecuente con el
uso de modelos tradicionales de educación. Sin
embargo, existen bases conceptuales en las asig-
naturas relacionadas con las ciencias básicas que
pueden tener un predominio teórico, pero que al
persistir en ellas, poco contribuyen a la formación
de habilidades del pensamiento superior como la
observación, el análisis, la inferencia, para que
contribuyan al desarrollo de competencias espe-
cíficas de la enfermera, encaminadas a la toma de
decisiones en contexto, para favorecer las perso-
nas y colectivos sujetos de cuidado.
Los estudiantes del programa de enfermería ob-
jeto de este estudio aplican los diferentes esti-
los de aprendizaje en los diversos semestres; sin
embargo, existe una tendencia a recurrir al estilo
de aprendizaje reflexivo y teórico, lo que con-
stituyen nuevos retos para los docentes, que van
desde un conocimiento no sólo del área especí-
fica objeto de enseñanza, hasta el fortalecimiento
de los diversos métodos, estrategias y recursos
didácticos, para propiciar en los estudiantes apre-
ndizajes significativos desde el aula.
-

Conclusiones
Los resultados de la investigación no evidenciaron
asociación estadística entre los estilos de apren-
dizaje y las variables sociodemográficas, perso-
nales y de recursos educativos; no obstante, aún
persiste un predominio del sexo femenino en la po-
blación que hace parte de la formación de la enfer-
mería como profesión, lo cual, concuerda con los
hallazgos referidos en otros estudios y puede estar
influenciado por rasgos culturales que han carac-
terizado el desarrollo histórico de la disciplina.
Los métodos didácticos utilizados por los docen-
tes, según ésta investigación, emplean en un alto
porcentaje la clase magistral, método que en los
primeros semestres de formación del pensamien-
to en la enfermería puede ser consecuente con el
uso de modelos tradicionales de educación. Sin
embargo, existen bases conceptuales en las asig-
naturas relacionadas con las ciencias básicas que
pueden tener un predominio teórico, pero que al
persistir en ellas, poco contribuyen a la formación
de habilidades del pensamiento superior como la
observación, el análisis, la inferencia, para que
contribuyan al desarrollo de competencias espe-
cíficas de la enfermera, encaminadas a la toma de
decisiones en contexto, para favorecer las perso-
nas y colectivos sujetos de cuidado.
Los estudiantes del programa de enfermería ob-
jeto de este estudio aplican los diferentes esti-
los de aprendizaje en los diversos semestres; sin
embargo, existe una tendencia a recurrir al estilo
de aprendizaje reflexivo y teórico, lo que con-
stituyen nuevos retos para los docentes, que van
desde un conocimiento no sólo del área especí-
fica objeto de enseñanza, hasta el fortalecimiento
de los diversos métodos, estrategias y recursos
didácticos, para propiciar en los estudiantes apre-
ndizajes significativos desde el aula.
-

Conclusiones
Los resultados de la investigación no evidenciaron
asociación estadística entre los estilos de apren-
dizaje y las variables sociodemográficas, perso-
nales y de recursos educativos; no obstante, aún
persiste un predominio del sexo femenino en la po-
blación que hace parte de la formación de la enfer-
mería como profesión, lo cual, concuerda con los
hallazgos referidos en otros estudios y puede estar
influenciado por rasgos culturales que han carac-
terizado el desarrollo histórico de la disciplina.
Los métodos didácticos utilizados por los docen-
tes, según ésta investigación, emplean en un alto
porcentaje la clase magistral, método que en los
primeros semestres de formación del pensamien-
to en la enfermería puede ser consecuente con el
uso de modelos tradicionales de educación. Sin
embargo, existen bases conceptuales en las asig-
naturas relacionadas con las ciencias básicas que
pueden tener un predominio teórico, pero que al
persistir en ellas, poco contribuyen a la formación
de habilidades del pensamiento superior como la
observación, el análisis, la inferencia, para que
contribuyan al desarrollo de competencias espe-
cíficas de la enfermera, encaminadas a la toma de
decisiones en contexto, para favorecer las perso-
nas y colectivos sujetos de cuidado.
Los estudiantes del programa de enfermería ob-
jeto de este estudio aplican los diferentes esti-
los de aprendizaje en los diversos semestres; sin
embargo, existe una tendencia a recurrir al estilo
de aprendizaje reflexivo y teórico, lo que con-
stituyen nuevos retos para los docentes, que van
desde un conocimiento no sólo del área especí-
fica objeto de enseñanza, hasta el fortalecimiento
de los diversos métodos, estrategias y recursos
didácticos, para propiciar en los estudiantes apre-
ndizajes significativos desde el aula.
-

Conclusiones
Los resultados de la investigación no evidenciaron
asociación estadística entre los estilos de apren-
dizaje y las variables sociodemográficas, perso-
nales y de recursos educativos; no obstante, aún
persiste un predominio del sexo femenino en la po-
blación que hace parte de la formación de la enfer-
mería como profesión, lo cual, concuerda con los
hallazgos referidos en otros estudios y puede estar
influenciado por rasgos culturales que han carac-
terizado el desarrollo histórico de la disciplina.
Los métodos didácticos utilizados por los docen-
tes, según ésta investigación, emplean en un alto
porcentaje la clase magistral, método que en los
primeros semestres de formación del pensamien-
to en la enfermería puede ser consecuente con el
uso de modelos tradicionales de educación. Sin
embargo, existen bases conceptuales en las asig-
naturas relacionadas con las ciencias básicas que
pueden tener un predominio teórico, pero que al
persistir en ellas, poco contribuyen a la formación
de habilidades del pensamiento superior como la
observación, el análisis, la inferencia, para que
contribuyan al desarrollo de competencias espe-
cíficas de la enfermera, encaminadas a la toma de
decisiones en contexto, para favorecer las perso-
nas y colectivos sujetos de cuidado.
Los estudiantes del programa de enfermería ob-
jeto de este estudio aplican los diferentes esti-
los de aprendizaje en los diversos semestres; sin
embargo, existe una tendencia a recurrir al estilo
de aprendizaje reflexivo y teórico, lo que con-
stituyen nuevos retos para los docentes, que van
desde un conocimiento no sólo del área especí-
fica objeto de enseñanza, hasta el fortalecimiento
de los diversos métodos, estrategias y recursos
didácticos, para propiciar en los estudiantes apre-
ndizajes significativos desde el aula.
-

Conclusiones
Los resultados de la investigación no evidenciaron
asociación estadística entre los estilos de apren-
dizaje y las variables sociodemográficas, perso-
nales y de recursos educativos; no obstante, aún
persiste un predominio del sexo femenino en la po-
blación que hace parte de la formación de la enfer-
mería como profesión, lo cual, concuerda con los
hallazgos referidos en otros estudios y puede estar
influenciado por rasgos culturales que han carac-
terizado el desarrollo histórico de la disciplina.
Los métodos didácticos utilizados por los docen-
tes, según ésta investigación, emplean en un alto
porcentaje la clase magistral, método que en los
primeros semestres de formación del pensamien-
to en la enfermería puede ser consecuente con el
uso de modelos tradicionales de educación. Sin
embargo, existen bases conceptuales en las asig-
naturas relacionadas con las ciencias básicas que
pueden tener un predominio teórico, pero que al
persistir en ellas, poco contribuyen a la formación
de habilidades del pensamiento superior como la
observación, el análisis, la inferencia, para que
contribuyan al desarrollo de competencias espe-
cíficas de la enfermera, encaminadas a la toma de
decisiones en contexto, para favorecer las perso-
nas y colectivos sujetos de cuidado.
Los estudiantes del programa de enfermería ob-
jeto de este estudio aplican los diferentes esti-
los de aprendizaje en los diversos semestres; sin
embargo, existe una tendencia a recurrir al estilo
de aprendizaje reflexivo y teórico, lo que con-
stituyen nuevos retos para los docentes, que van
desde un conocimiento no sólo del área especí-
fica objeto de enseñanza, hasta el fortalecimiento
de los diversos métodos, estrategias y recursos
didácticos, para propiciar en los estudiantes apre-
ndizajes significativos desde el aula.
-

Conclusiones
Los resultados de la investigación no evidenciaron
asociación estadística entre los estilos de apren-
dizaje y las variables sociodemográficas, perso-
nales y de recursos educativos; no obstante, aún
persiste un predominio del sexo femenino en la po-
blación que hace parte de la formación de la enfer-
mería como profesión, lo cual, concuerda con los
hallazgos referidos en otros estudios y puede estar
influenciado por rasgos culturales que han carac-
terizado el desarrollo histórico de la disciplina.
Los métodos didácticos utilizados por los docen-
tes, según ésta investigación, emplean en un alto
porcentaje la clase magistral, método que en los
primeros semestres de formación del pensamien-
to en la enfermería puede ser consecuente con el
uso de modelos tradicionales de educación. Sin
embargo, existen bases conceptuales en las asig-
naturas relacionadas con las ciencias básicas que
pueden tener un predominio teórico, pero que al
persistir en ellas, poco contribuyen a la formación
de habilidades del pensamiento superior como la
observación, el análisis, la inferencia, para que
contribuyan al desarrollo de competencias espe-
cíficas de la enfermera, encaminadas a la toma de
decisiones en contexto, para favorecer las perso-
nas y colectivos sujetos de cuidado.
Los estudiantes del programa de enfermería ob-
jeto de este estudio aplican los diferentes esti-
los de aprendizaje en los diversos semestres; sin
embargo, existe una tendencia a recurrir al estilo
de aprendizaje reflexivo y teórico, lo que con-
stituyen nuevos retos para los docentes, que van
desde un conocimiento no sólo del área especí-
fica objeto de enseñanza, hasta el fortalecimiento
de los diversos métodos, estrategias y recursos
didácticos, para propiciar en los estudiantes apre-
ndizajes significativos desde el aula.
-

Conclusiones
Los resultados de la investigación no evidenciaron
asociación estadística entre los estilos de apren-
dizaje y las variables sociodemográficas, perso-
nales y de recursos educativos; no obstante, aún
persiste un predominio del sexo femenino en la po-
blación que hace parte de la formación de la enfer-
mería como profesión, lo cual, concuerda con los
hallazgos referidos en otros estudios y puede estar
influenciado por rasgos culturales que han carac-
terizado el desarrollo histórico de la disciplina.
Los métodos didácticos utilizados por los docen-
tes, según ésta investigación, emplean en un alto
porcentaje la clase magistral, método que en los
primeros semestres de formación del pensamien-
to en la enfermería puede ser consecuente con el
uso de modelos tradicionales de educación. Sin
embargo, existen bases conceptuales en las asig-
naturas relacionadas con las ciencias básicas que
pueden tener un predominio teórico, pero que al
persistir en ellas, poco contribuyen a la formación
de habilidades del pensamiento superior como la
observación, el análisis, la inferencia, para que
contribuyan al desarrollo de competencias espe-
cíficas de la enfermera, encaminadas a la toma de
decisiones en contexto, para favorecer las perso-
nas y colectivos sujetos de cuidado.
Los estudiantes del programa de enfermería ob-
jeto de este estudio aplican los diferentes esti-
los de aprendizaje en los diversos semestres; sin
embargo, existe una tendencia a recurrir al estilo
de aprendizaje reflexivo y teórico, lo que con-
stituyen nuevos retos para los docentes, que van
desde un conocimiento no sólo del área especí-
fica objeto de enseñanza, hasta el fortalecimiento
de los diversos métodos, estrategias y recursos
didácticos, para propiciar en los estudiantes apre-
ndizajes significativos desde el aula.
-

Conclusiones
Los resultados de la investigación no evidenciaron
asociación estadística entre los estilos de apren-
dizaje y las variables sociodemográficas, perso-
nales y de recursos educativos; no obstante, aún
persiste un predominio del sexo femenino en la po-
blación que hace parte de la formación de la enfer-
mería como profesión, lo cual, concuerda con los
hallazgos referidos en otros estudios y puede estar
influenciado por rasgos culturales que han carac-
terizado el desarrollo histórico de la disciplina.
Los métodos didácticos utilizados por los docen-
tes, según ésta investigación, emplean en un alto
porcentaje la clase magistral, método que en los
primeros semestres de formación del pensamien-
to en la enfermería puede ser consecuente con el
uso de modelos tradicionales de educación. Sin
embargo, existen bases conceptuales en las asig-
naturas relacionadas con las ciencias básicas que
pueden tener un predominio teórico, pero que al
persistir en ellas, poco contribuyen a la formación
de habilidades del pensamiento superior como la
observación, el análisis, la inferencia, para que
contribuyan al desarrollo de competencias espe-
cíficas de la enfermera, encaminadas a la toma de
decisiones en contexto, para favorecer las perso-
nas y colectivos sujetos de cuidado.
Los estudiantes del programa de enfermería ob-
jeto de este estudio aplican los diferentes esti-
los de aprendizaje en los diversos semestres; sin
embargo, existe una tendencia a recurrir al estilo
de aprendizaje reflexivo y teórico, lo que con-
stituyen nuevos retos para los docentes, que van
desde un conocimiento no sólo del área especí-
fica objeto de enseñanza, hasta el fortalecimiento
de los diversos métodos, estrategias y recursos
didácticos, para propiciar en los estudiantes apre-
ndizajes significativos desde el aula.
-

Conclusiones
Los resultados de la investigación no evidenciaron
asociación estadística entre los estilos de apren-
dizaje y las variables sociodemográficas, perso-
nales y de recursos educativos; no obstante, aún
persiste un predominio del sexo femenino en la po-
blación que hace parte de la formación de la enfer-
mería como profesión, lo cual, concuerda con los
hallazgos referidos en otros estudios y puede estar
influenciado por rasgos culturales que han carac-
terizado el desarrollo histórico de la disciplina.
Los métodos didácticos utilizados por los docen-
tes, según ésta investigación, emplean en un alto
porcentaje la clase magistral, método que en los
primeros semestres de formación del pensamien-
to en la enfermería puede ser consecuente con el
uso de modelos tradicionales de educación. Sin
embargo, existen bases conceptuales en las asig-
naturas relacionadas con las ciencias básicas que
pueden tener un predominio teórico, pero que al
persistir en ellas, poco contribuyen a la formación
de habilidades del pensamiento superior como la
observación, el análisis, la inferencia, para que
contribuyan al desarrollo de competencias espe-
cíficas de la enfermera, encaminadas a la toma de
decisiones en contexto, para favorecer las perso-
nas y colectivos sujetos de cuidado.
Los estudiantes del programa de enfermería ob-
jeto de este estudio aplican los diferentes esti-
los de aprendizaje en los diversos semestres; sin
embargo, existe una tendencia a recurrir al estilo
de aprendizaje reflexivo y teórico, lo que con-
stituyen nuevos retos para los docentes, que van
desde un conocimiento no sólo del área especí-
fica objeto de enseñanza, hasta el fortalecimiento
de los diversos métodos, estrategias y recursos
didácticos, para propiciar en los estudiantes apre-
ndizajes significativos desde el aula.
-

Conclusiones
Los resultados de la investigación no evidenciaron
asociación estadística entre los estilos de apren-
dizaje y las variables sociodemográficas, perso-
nales y de recursos educativos; no obstante, aún
persiste un predominio del sexo femenino en la po-
blación que hace parte de la formación de la enfer-
mería como profesión, lo cual, concuerda con los
hallazgos referidos en otros estudios y puede estar
influenciado por rasgos culturales que han carac-
terizado el desarrollo histórico de la disciplina.
Los métodos didácticos utilizados por los docen-
tes, según ésta investigación, emplean en un alto
porcentaje la clase magistral, método que en los
primeros semestres de formación del pensamien-
to en la enfermería puede ser consecuente con el
uso de modelos tradicionales de educación. Sin
embargo, existen bases conceptuales en las asig-
naturas relacionadas con las ciencias básicas que
pueden tener un predominio teórico, pero que al
persistir en ellas, poco contribuyen a la formación
de habilidades del pensamiento superior como la
observación, el análisis, la inferencia, para que
contribuyan al desarrollo de competencias espe-
cíficas de la enfermera, encaminadas a la toma de
decisiones en contexto, para favorecer las perso-
nas y colectivos sujetos de cuidado.
Los estudiantes del programa de enfermería ob-
jeto de este estudio aplican los diferentes esti-
los de aprendizaje en los diversos semestres; sin
embargo, existe una tendencia a recurrir al estilo
de aprendizaje reflexivo y teórico, lo que con-
stituyen nuevos retos para los docentes, que van
desde un conocimiento no sólo del área especí-
fica objeto de enseñanza, hasta el fortalecimiento
de los diversos métodos, estrategias y recursos
didácticos, para propiciar en los estudiantes apre-
ndizajes significativos desde el aula.
-

Conclusiones
Los resultados de la investigación no evidenciaron
asociación estadística entre los estilos de apren-
dizaje y las variables sociodemográficas, perso-
nales y de recursos educativos; no obstante, aún
persiste un predominio del sexo femenino en la po-
blación que hace parte de la formación de la enfer-
mería como profesión, lo cual, concuerda con los
hallazgos referidos en otros estudios y puede estar
influenciado por rasgos culturales que han carac-
terizado el desarrollo histórico de la disciplina.
Los métodos didácticos utilizados por los docen-
tes, según ésta investigación, emplean en un alto
porcentaje la clase magistral, método que en los
primeros semestres de formación del pensamien-
to en la enfermería puede ser consecuente con el
uso de modelos tradicionales de educación. Sin
embargo, existen bases conceptuales en las asig-
naturas relacionadas con las ciencias básicas que
pueden tener un predominio teórico, pero que al
persistir en ellas, poco contribuyen a la formación
de habilidades del pensamiento superior como la
observación, el análisis, la inferencia, para que
contribuyan al desarrollo de competencias espe-
cíficas de la enfermera, encaminadas a la toma de
decisiones en contexto, para favorecer las perso-
nas y colectivos sujetos de cuidado.
Los estudiantes del programa de enfermería ob-
jeto de este estudio aplican los diferentes esti-
los de aprendizaje en los diversos semestres; sin
embargo, existe una tendencia a recurrir al estilo
de aprendizaje reflexivo y teórico, lo que con-
stituyen nuevos retos para los docentes, que van
desde un conocimiento no sólo del área especí-
fica objeto de enseñanza, hasta el fortalecimiento
de los diversos métodos, estrategias y recursos
didácticos, para propiciar en los estudiantes apre-
ndizajes significativos desde el aula.
-

Conclusiones
Los resultados de la investigación no evidenciaron
asociación estadística entre los estilos de apren-
dizaje y las variables sociodemográficas, perso-
nales y de recursos educativos; no obstante, aún
persiste un predominio del sexo femenino en la po-
blación que hace parte de la formación de la enfer-
mería como profesión, lo cual, concuerda con los
hallazgos referidos en otros estudios y puede estar
influenciado por rasgos culturales que han carac-
terizado el desarrollo histórico de la disciplina.
Los métodos didácticos utilizados por los docen-
tes, según ésta investigación, emplean en un alto
porcentaje la clase magistral, método que en los
primeros semestres de formación del pensamien-
to en la enfermería puede ser consecuente con el
uso de modelos tradicionales de educación. Sin
embargo, existen bases conceptuales en las asig-
naturas relacionadas con las ciencias básicas que
pueden tener un predominio teórico, pero que al
persistir en ellas, poco contribuyen a la formación
de habilidades del pensamiento superior como la
observación, el análisis, la inferencia, para que
contribuyan al desarrollo de competencias espe-
cíficas de la enfermera, encaminadas a la toma de
decisiones en contexto, para favorecer las perso-
nas y colectivos sujetos de cuidado.
Los estudiantes del programa de enfermería ob-
jeto de este estudio aplican los diferentes esti-
los de aprendizaje en los diversos semestres; sin
embargo, existe una tendencia a recurrir al estilo
de aprendizaje reflexivo y teórico, lo que con-
stituyen nuevos retos para los docentes, que van
desde un conocimiento no sólo del área especí-
fica objeto de enseñanza, hasta el fortalecimiento
de los diversos métodos, estrategias y recursos
divas estudiantes apre-
autoses desde el aula.
-

Conclusiones
Los resultados de la investigación no evidenciaron
asociación estadística entre los estilos de apren-
dizaje y las variables sociodemográficas, perso-
nales y de recursos educativos; no obstante, aún
persiste un predominio del sexo femenino en la po-
blación que hace parte de la formación de la enfer-
mería como profesión, lo cual, concuerda con los
hallazgos referidos en otros estudios y puede estar
influenciado por rasgos culturales que han carac-
terizado el desarrollo histórico de la disciplina.
Los métodos didácticos utilizados por los docen-
tes, según ésta investigación, emplean en un alto
porcentaje la clase magistral, método que en los
primeros semestres de formación del pensamien-
to en la enfermería puede ser consecuente con el
uso de modelos tradicionales de educación. Sin
embargo, existen bases conceptuales en las asig-
naturas relacionadas con las ciencias básicas que
pueden tener un predominio teórico, pero que al
persistir en ellas, poco contribuyen a la formación
de habilidades del pensamiento superior como la
observación, el análisis, la inferencia, para que
contribuyan al desarrollo de competencias espe-
cíficas de la enfermera, encaminadas a la toma de
decisiones en contexto, para favorecer las perso-
nas y colectivos sujetos de cuidado.
Los estudiantes del programa de enfermería ob-
jeto de este estudio aplican los diferentes esti-
los de aprendizaje en los diversos semestres; sin
embargo, existe una tendencia a recurrir al estilo
de aprendizaje reflexivo y teórico, lo que con-
stituyen nuevos retos para los docentes, que van
desde un conocimiento no sólo del área especí-
fica objeto de enseñanza, hasta el fortalecimiento
de los diversos métodos, estrategias y recursos
didácticos, para propiciar en los estudiantes apre-
ndizajes significativos desde el aula.
-

Conclusiones
Los resultados de la investigación no evidenciaron
asociación estadística entre los estilos de apren-
dizaje y las variables sociodemográficas, perso-
nales y de recursos educativos; no obstante, aún
persiste un predominio del sexo femenino en la po-
blación que hace parte de la formación de la enfer-
mería como profesión, lo cual, concuerda con los
hallazgos referidos en otros estudios y puede estar
influenciado por rasgos culturales que han carac-
terizado el desarrollo histórico de la disciplina.
Los métodos didácticos utilizados por los docen-
tes, según ésta investigación, emplean en un alto
porcentaje la clase magistral, método que en los
primeros semestres de formación del pensamien-
to en la enfermería puede ser consecuente con el
uso de modelos tradicionales de educación. Sin
embargo, existen bases conceptuales en las asig-
naturas relacionadas con las ciencias básicas que
pueden tener un predominio teórico, pero que al
persistir en ellas, poco contribuyen a la formación
de habilidades del pensamiento superior como la
observación, el análisis, la inferencia, para que
contribuyan al desarrollo de competencias espe-
cíficas de la enfermera, encaminadas a la toma de
decisiones en contexto, para favorecer las perso-
nas y colectivos sujetos de cuidado.
Los estudiantes del programa de enfermería ob-
jeto de este estudio aplican los diferentes esti-
los de aprendizaje en los diversos semestres; sin
embargo, existe una tendencia a recurrir al estilo
de aprendizaje reflexivo y teórico, lo que con-
stituyen nuevos retos para los docentes, que van
desde un conocimiento no sólo del área especí-
fica objeto de enseñanza, hasta el fortalecimiento
de los diversos métodos, estrategias y recursos
didácticos, para propiciar en los estudiantes apre-
ndizajes significativos desde el aula.
-

Conclusiones
Los resultados de la investigación no evidenciaron
asociación estadística entre los estilos de apren-
dizaje y las variables sociodemográficas, perso-
nales y de recursos educativos; no obstante, aún
persiste un predominio del sexo femenino en la po-
blación que hace parte de la formación de la enfer-
mería como profesión, lo cual, concuerda con los
hallazgos referidos en otros estudios y puede estar
influenciado por rasgos culturales que han carac-
terizado el desarrollo histórico de la disciplina.
Los métodos didácticos utilizados por los docen-
tes, según ésta investigación, emplean en un alto
porcentaje la clase magistral, método que en los
primeros semestres de formación del pensamien-
to en la enfermería puede ser consecuente con el
uso de modelos tradicionales de educación. Sin
embargo, existen bases conceptuales en las asig-
naturas relacionadas con las ciencias básicas que
pueden tener un predominio teórico, pero que al
persistir en ellas, poco contribuyen a la formación
de habilidades del pensamiento superior como la
observación, el análisis, la inferencia, para que
contribuyan al desarrollo de competencias espe-
cíficas de la enfermera, encaminadas a la toma de
decisiones en contexto, para favorecer las perso-
nas y colectivos sujetos de cuidado.
Los estudiantes del programa de enfermería ob-
jeto de este estudio aplican los diferentes esti-
los de aprendizaje en los diversos semestres; sin
embargo, existe una tendencia a recurrir al estilo
de aprendizaje reflexivo y teórico, lo que con-
stituyen nuevos retos para los docentes, que van
desde un conocimiento no sólo del área especí-
fica objeto de enseñanza, hasta el fortalecimiento
de los diversos métodos, estrategias y recursos
didácticos, para propiciar en los estudiantes apre-
ndizajes significativos desde el aula.
-

Conclusiones
Los resultados de la investigación no evidenciaron
asociación estadística entre los estilos de apren-
dizaje y las variables sociodemográficas, perso-
nales y de recursos educativos; no obstante, aún
persiste un predominio del sexo femenino en la po-
blación que hace parte de la formación de la enfer-
mería como profesión, lo cual, concuerda con los
hallazgos referidos en otros estudios y puede estar
influenciado por rasgos culturales que han carac-
terizado el desarrollo histórico de la disciplina.
Los métodos didácticos utilizados por los docen-
tes, según ésta investigación, emplean en un alto
porcentaje la clase magistral, método que en los
primeros semestres de formación del pensamien-
to en la enfermería puede ser consecuente con el
uso de modelos tradicionales de educación. Sin
embargo, existen bases conceptuales en las asig-
naturas relacionadas con las ciencias básicas que
pueden tener un predominio teórico, pero que al
persistir en ellas, poco contribuyen a la formación
de habilidades del pensamiento superior como la
observación, el análisis, la inferencia, para que
contribuyan al desarrollo de competencias espe-
cíficas de la enfermera, encaminadas a la toma de
decisiones en contexto, para favorecer las perso-
nas y colectivos sujetos de cuidado.
Los estudiantes del programa de enfermería ob-
jeto de este estudio aplican los diferentes esti-
los de aprendizaje en los diversos semestres; sin
embargo, existe una tendencia a recurrir al estilo
de aprendizaje reflexivo y teórico, lo que con-
stituyen nuevos retos para los docentes, que van
desde un conocimiento no sólo del área especí-
fica objeto de enseñanza, hasta el fortalecimiento
de los diversos métodos, estrategias y recursos
didácticos, para propiciar en los estudiantes apre-
ndizajes significativos desde el aula.
-

Conclusiones
Los resultados de la investigación no evidenciaron
asociación estadística entre los estilos de apren-
dizaje y las variables sociodemográficas, perso-
nales y de recursos educativos; no obstante, aún
persiste un predominio del sexo femenino en la po-
blación que hace parte de la formación de la enfer-
mería como profesión, lo cual, concuerda con los
hallazgos referidos en otros estudios y puede estar
influenciado por rasgos culturales que han carac-
terizado el desarrollo histórico de la disciplina.
Los métodos didácticos utilizados por los docen-
tes, según ésta investigación, emplean en un alto
porcentaje la clase magistral, método que en los
primeros semestres de formación del pensamien-
to en la enfermería puede ser consecuente con el
uso de modelos tradicionales de educación. Sin
embargo, existen bases conceptuales en las asig-
naturas relacionadas con las ciencias básicas que
pueden tener un predominio teórico, pero que al
persistir en ellas, poco contribuyen a la formación
de habilidades del pensamiento superior como la
observación, el análisis, la inferencia, para que
contribuyan al desarrollo de competencias espe-
cíficas de la enfermera, encaminadas a la toma de
decisiones en contexto, para favorecer las perso-
nas y colectivos sujetos de cuidado.
Los estudiantes del programa de enfermería ob-
jeto de este estudio aplican los diferentes esti-
los de aprendizaje en los diversos semestres; sin
embargo, existe una tendencia a recurrir al estilo
de aprendizaje reflexivo y teórico, lo que con-
stituyen nuevos retos para los docentes, que van
desde un conocimiento no sólo del área especí-
fica objeto de enseñanza, hasta el fortalecimiento
de los diversos métodos, estrategias y recursos
didácticos, para propiciar en los estudiantes apre-
ndizajes significativos desde el aula.
-

Conclusiones
Los resultados de la investigación no evidenciaron
asociación estadística entre los estilos de apren-
dizaje y las variables sociodemográficas, perso-
nales y de recursos educativos; no obstante, aún
persiste un predominio del sexo femenino en la po-
blación que hace parte de la formación de la enfer-
mería como profesión, lo cual, concuerda con los
hallazgos referidos en otros estudios y puede estar
influenciado por rasgos culturales que han carac-
terizado el desarrollo histórico de la disciplina.
Los métodos didácticos utilizados por los docen-
tes, según ésta investigación, emplean en un alto
porcentaje la clase magistral, método que en los
primeros semestres de formación del pensamien-
to en la enfermería puede ser consecuente con el
uso de modelos tradicionales de educación. Sin
embargo, existen bases conceptuales en las asig-
naturas relacionadas con las ciencias básicas que
pueden tener un predominio teórico, pero que al
persistir en ellas, poco contribuyen a la formación
de habilidades del pensamiento superior como la
observación, el análisis, la inferencia, para que
contribuyan al desarrollo de competencias espe-
cíficas de la enfermera, encaminadas a la toma de
decisiones en contexto, para favorecer las perso-
nas y colectivos sujetos de cuidado.
Los estudiantes del programa de enfermería ob-
jeto de este estudio aplican los diferentes esti-
los de aprendizaje en los diversos semestres; sin
embargo, existe una tendencia a recurrir al estilo
de aprendizaje reflexivo y teórico, lo que con-
stituyen nuevos retos para los docentes, que van
desde un conocimiento no sólo del área especí-
fica objeto de enseñanza, hasta el fortalecimiento
de los diversos métodos, estrategias y recursos
didácticos, para propiciar en los estudiantes apre-
ndizajes significativos desde el aula.
-

Conclusiones
Los resultados de la investigación no evidenciaron
asociación estadística entre los estilos de apren-
dizaje y las variables sociodemográficas, perso-
nales y de recursos educativos; no obstante, aún
persiste un predominio del sexo femenino en la po-
blación que hace parte de la formación de la enfer-
mería como profesión, lo cual, concuerda con los
hallazgos referidos en otros estudios y puede estar
influenciado por rasgos culturales que han carac-
terizado el desarrollo histórico de la disciplina.
Los métodos didácticos utilizados por los docen-
tes, según ésta investigación, emplean en un alto
porcentaje la clase magistral, método que en los
primeros semestres de formación del pensamien-
to en la enfermería puede ser consecuente con el
uso de modelos tradicionales de educación. Sin
embargo, existen bases conceptuales en las asig-
naturas relacionadas con las ciencias básicas que
pueden tener un predominio teórico, pero que al
persistir en ellas, poco contribuyen a la formación
de habilidades del pensamiento superior como la
observación, el análisis, la inferencia, para que
contribuyan al desarrollo de competencias espe-
cíficas de la enfermera, encaminadas a la toma de
decisiones en contexto, para favorecer las perso-
nas y colectivos sujetos de cuidado.
Los estudiantes del programa de enfermería ob-
jeto de este estudio aplican los diferentes esti-
los de aprendizaje en los diversos semestres; sin
embargo, existe una tendencia a recurrir al estilo
de aprendizaje reflexivo y teórico, lo que con-
stituyen nuevos retos para los docentes, que van
desde un conocimiento no sólo del área especí-
fica objeto de enseñanza, hasta el fortalecimiento
de los diversos métodos, estrategias y recursos
didácticos, para propiciar en los estudiantes apre-
ndizajes significativos desde el aula.
-

Conclusiones
Los resultados de la investigación no evidenciaron
asociación estadística entre los estilos de apren-
dizaje y las variables sociodemográficas, perso-
nales y de recursos educativos; no obstante, aún
persiste un predominio del sexo femenino en la po-
blación que hace parte de la formación de la enfer-
mería como profesión, lo cual, concuerda con los
hallazgos referidos en otros estudios y puede estar
influenciado por rasgos culturales que han carac-
terizado el desarrollo histórico de la disciplina.
Los métodos didácticos utilizados por los docen-
tes, según ésta investigación, emplean en un alto
porcentaje la clase magistral, método que en los
primeros semestres de formación del pensamien-
to en la enfermería puede ser consecuente con el
uso de modelos tradicionales de educación. Sin
embargo, existen bases conceptuales en las asig-
naturas relacionadas con las ciencias básicas que
pueden tener un predominio teórico, pero que al
persistir en ellas, poco contribuyen a la formación
de habilidades del pensamiento superior como la
observación, el análisis, la inferencia, para que
contribuyan al desarrollo de competencias espe-
cíficas de la enfermera, encaminadas a la toma de
decisiones en contexto, para favorecer las perso-
nas y colectivos sujetos de cuidado.
Los estudiantes del programa de enfermería ob-
jeto de este estudio aplican los diferentes esti-
los de aprendizaje en los diversos semestres; sin
embargo, existe una tendencia a recurrir al estilo
de aprendizaje reflexivo y teórico, lo que con-
stituyen nuevos retos para los docentes, que van
desde un conocimiento no sólo del área especí-
fica objeto de enseñanza, hasta el fortalecimiento
de los diversos métodos, estrategias y recursos
didácticos, para propiciar en los estudiantes apre-
ndizajes significativos desde el aula.
-

Conclusiones
Los resultados de la investigación no evidenciaron
asociación estadística entre los estilos de apren-
dizaje y las variables sociodemográficas, perso-
nales y de recursos educativos; no obstante, aún
persiste un predominio del sexo femenino en la po-
blación que hace parte de la formación de la enfer-
mería como profesión, lo cual, concuerda con los
hallazgos referidos en otros estudios y puede estar
influenciado por rasgos culturales que han carac-
terizado el desarrollo histórico de la disciplina.
Los métodos didácticos utilizados por los docen-
tes, según ésta investigación, emplean en un alto
porcentaje la clase magistral, método que en los
primeros semestres de formación del pensamien-
to en la enfermería puede ser consecuente con el
uso de modelos tradicionales de educación. Sin
embargo, existen bases conceptuales en las asig-
naturas relacionadas con las ciencias básicas que
pueden tener un predominio teórico, pero que al
persistir en ellas, poco contribuyen a la formación
de habilidades del pensamiento superior como la
observación, el análisis, la inferencia, para que
contribuyan al desarrollo de competencias espe-
cíficas de la enfermera, encaminadas a la toma de
decisiones en contexto, para favorecer las perso-
nas y colectivos sujetos de cuidado.
Los estudiantes del programa de enfermería ob-
jeto de este estudio aplican los diferentes esti-
los de aprendizaje en los diversos semestres; sin
embargo, existe una tendencia a recurrir al estilo
de aprendizaje reflexivo y teórico, lo que con-
stituyen nuevos retos para los docentes, que van
desde un conocimiento no sólo del área especí-
fica objeto de enseñanza, hasta el fortalecimiento
de los diversos métodos, estrategias y recursos
didácticos, para propiciar en los estudiantes apre-
ndizajes significativos desde el aula.
-

Conclusiones
Los resultados de la investigación no evidenciaron
asociación estadística entre los estilos de apren-
dizaje y las variables sociodemográficas, perso-
nales y de recursos educativos; no obstante, aún
persiste un predominio del sexo femenino en la po-
blación que hace parte de la formación de la enfer-
mería como profesión, lo cual, concuerda con los
hallazgos referidos en otros estudios y puede estar
influenciado por rasgos culturales que han carac-
terizado el desarrollo histórico de la disciplina.
Los métodos didácticos utilizados por los docen-
tes, según ésta investigación, emplean en un alto
porcentaje la clase magistral, método que en los
primeros semestres de formación del pensamien-
to en la enfermería puede ser consecuente con el
uso de modelos tradicionales de educación. Sin
embargo, existen bases conceptuales en las asig-
naturas relacionadas con las ciencias básicas que
pueden tener un predominio teórico, pero que al
persistir en ellas, poco contribuyen a la formación
de habilidades del pensamiento superior como la
observación, el análisis, la inferencia, para que
contribuyan al desarrollo de competencias espe-
cíficas de la enfermera, encaminadas a la toma de
decisiones en contexto, para favorecer las perso-
nas y colectivos sujetos de cuidado.
Los estudiantes del programa de enfermería ob-
jeto de este estudio aplican los diferentes esti-
los de aprendizaje en los diversos semestres; sin
embargo, existe una tendencia a recurrir al estilo
de aprendizaje reflexivo y teórico, lo que con-
stituyen nuevos retos para los docentes, que van
desde un conocimiento no sólo del área especí-
fica objeto de enseñanza, hasta el fortalecimiento
de los diversos métodos, estrategias y recursos
didácticos, para propiciar en los estudiantes apre-
ndizajes significativos desde el aula.
-

Conclusiones
Los resultados de la investigación no evidenciaron
asociación estadística entre los estilos de apren-
dizaje y las variables sociodemográficas, perso-
nales y de recursos educativos; no obstante, aún
persiste un predominio del sexo femenino en la po-
blación que hace parte de la formación de la enfer-
mería como profesión, lo cual, concuerda con los
hallazgos referidos en otros estudios y puede estar
influenciado por rasgos culturales que han carac-
terizado el desarrollo histórico de la disciplina.
Los métodos didácticos utilizados por los docen-
tes, según ésta investigación, emplean en un alto
porcentaje la clase magistral, método que en los
primeros semestres de formación del pensamien-
to en la enfermería puede ser consecuente con el
uso de modelos tradicionales de educación. Sin
embargo, existen bases conceptuales en las asig-
naturas relacionadas con las ciencias básicas que
pueden tener un predominio teórico, pero que al
persistir en ellas, poco contribuyen a la formación
de habilidades del pensamiento superior como la
observación, el análisis, la inferencia, para que
contribuyan al desarrollo de competencias espe-
cíficas de la enfermera, encaminadas a la toma de
decisiones en contexto, para favorecer las perso-
nas y colectivos sujetos de cuidado.
Los estudiantes del programa de enfermería ob-
jeto de este estudio aplican los diferentes esti-
los de aprendizaje en los diversos semestres; sin
embargo, existe una tendencia a recurrir al estilo
de aprendizaje reflexivo y teórico, lo que con-
stituyen nuevos retos para los docentes, que van
desde un conocimiento no sólo del área especí-
fica objeto de enseñanza, hasta el fortalecimiento
de los diversos métodos, estrategias y recursos
didácticos, para propiciar en los estudiantes apre-
ndizajes significativos desde el aula.
Conflicto de Intereses

Las autoras declaran no tener ningún conflicto de intereses en la investigación realizada. 
ISSN-PRINT

$1794-9831$

E-ISSN 2322-7028

Vol. 16 No. 2

May - Ago 2019

Cúcuta, Colombia

\section{Referencias bibliográficas}

1. González F, Sáez K, Ramírez J. Perfiles de estilos de aprendizaje y rendimiento académico en estudiantes de primer año de enfermería. Cienc Enferm. 2016;22(1):87-99.

2. González Chordá VM, Maciá Soler ML. Evaluation of the quality of the teaching-learning process in undergraduate courses in Nursing. Rev Lat Am Enfermagem. 2015;23(4):700-7.

3. Isaza Valencia L. Estilos de Aprendizaje: una apuesta por el desempeño académico de los estudiantes en la Educación Superior. Rev Encuentros [Internet]. 14 de noviembre de 2014 [citado 8 de junio de 2018];12(2). Disponible en: http://ojs.uac.edu.co/index.php/encuentros/article/view/267

4. Gordón F del RA. Implicaciones del conocimiento de la teoría de los estilos de aprendizaje en el ejercicio profesional del docente universitario. J Learn Styles [Internet]. 31 de octubre de 2016 [citado 8 de junio de 2018];9(18). Disponible en: http://learningstyles.uvu.edu/index.php/jls/article/view/298

5. Bayona Montaño LA, Campo Torregrosa Y. Estrategias de aprendizaje en relación con el rendimiento académico en estudiantes de enfermería. Ánfora [Internet]. 2014 [citado 8 junio 2018] ;21(36):149163. Disponible en: http://www.redalyc.org/articulo.oa? id=357833887006.

6. Yancen Tinoco LM, Consuegra Cabally D, Herrera González K, Pacheco Siado B, Díaz Mass D. Education strategies used by the teacher of the Nursing Program in a University in the city of Barranquilla (Colombia) versus the student's learning styles in this Program. 2013; 29(3):405-416.

7. Durán Aponte E, Pujol L. Propiedades psicométricas del cuestionario de estilos de aprendizaje (CHAEA) en estudiantes de carreras técnicas. Revista Educación y Futuro Digital. 2012; 3: 61-70.

8. Gómez Bustamante EM, Jaimes Morales J, Severiche Sierra C. Estilos de aprendizaje en universitarios, modalidad de educación a distancia. Revista Virtual Universidad Católica del Norte. 2017; 50: 383-393

9. Universidad Nacional de Colombia: GAIA - Grupo de Ambientes Inteligentes Adaptativos [Internet]. [citado 8 de junio de 2018]. Disponible en: http://froac.manizales.unal.edu.co/chaea/test.php

10. Hoffman AF, Liporace MF. Cuestionario Honey-Alonso de estilos de aprendizaje: Análisis de sus propiedades Psicométricas en Estudiantes Universitarios. 2013; 10(1).

11. Rodríguez MDR, Sanmiguel Salazar M, Jimenez Villarreal J, Esparza Olage R. Análisis de los estilos de aprendizaje en estudiantes universitarios del área de la salud. Rev de estilos de aprendizaje. 9(17):21.

12. Villalba AB. Estilos de aprendizaje en alumnos universitarios de Profesorado en Biología y Licenciatura en Biodiversidad. J Learn Styles [Internet]. 31 de octubre de 2015 [citado 8 de junio de 2018];8(16). Disponible en: http://learningstyles.uvu.edu/index.php/jls/article/view/241

13. Alducin Ochoa JM, Vázquez Martínez AI. Estilos de aprendizaje, variables sociodemográficas y rendimiento académico en estudiantes de Ingeniería de Edificación. Rev Electrónica Educ. 2016;21(1):1.

14. Collière MF. Promover la vida. 2da ed. México: McGraw Hill Educación; 2009. 350 p.

15. Carrera IDC, Gutiérrez Nieto M, Barón López FJ, Labajos Manzanares MT. Influencia del nivel educativo de los padres en el rendimiento académico, las estrategias de aprendizaje y los estilos de aprendizaje desde la perspectiva de género. Journal of Learning Style. 2014;7(13):64-84.

16. Talbot AL, Dorrian J, Chapman J. Using the Theory of Planned Behaviour to examine enrolled nursing students' intention to care for patients with alcohol dependence: A survey study. Nurse Educ Today. 2015;35(11):1054-61.

17. García Sánchez M del R, Reyes Añorve J, Godínez Alarcón G. Las Tic en la educación superior, innovaciones y retos / The ICT in higher education, innovations and challenges. RICSH Rev Iberoam Las Cienc Soc Humanísticas [Internet]. 4 de enero de 2018 [citado 8 de junio de 2018];6(12). Disponible en: http://www.ricsh.org.mx/index.php/RICSH/article/view/135

18. Sander $P$. La investigación sobre nuestros alumnos, en pro de una mayor eficacia en la enseñanza uni- 
versitaria. Electronic Journal of Research in Education Psychology 3.5 2017;3(5):113-130.

19. Hernández R. La estrategia didáctica frente a los estilos de aprendizaje en la educación superior. Educación Médica 19.1 2018; 19(1):2-8.

20. Vizeshfar F, Torabizadeh $C$. The effect of teaching based on dominant learning style on nursing students' academic achievement. Nurse Educ Pract. 2018;28:103-8.

21. Medina Moya JLM, Jarauta Borrasca BJ, Imbernon Muñoz FI. Cuadernos de docencia universitaria 17. La enseñanza reflexiva en la educación superior. Barcelona: Octaedro; 43 p.

22. Benner P. From Novice to Expert. Excellence and Power in Clinical Nursing Practice. New Jersey: Prentice Hall Health; 1980. 307 p.

23. Benner P, Sutphen M, Leonard V DL. Educating Nurses. A call for radical transformation. Stanford. Estados Unidos de América: Jossey- Bass; 2010. 249 p.

24. Stirling BV, Alquraini WA. Using VARK to assess Saudi nursing students' learning style preferences: Do they differ from other health professionals? J Taibah Univ Med Sci. 2017;12(2):125-30.

25. Olivos P, Santos A, Martín S, Cañas M, Gómez Lázaro E, Maya Y. The relationship between learning styles and motivation to transfer of learning in a vocational training programme. Suma Psicológica. 2016;23(1):25-32.

26. García Luna AJ, Tamez Herrera C, Lozano Rodríguez A. Estilos de aprendizaje y rendimiento académico en alumnos de segundo grado de secundaria. J Learn Styles [Internet]. 30 de abril de 2015 [citado 8 de junio de 2018];8(15). Disponible en: http://learningstyles.uvu.edu/index.php/jls/article/view/230

27. Hernandez Serrano M, Serate Gonzales S, Campos Ortuño R. Influencia del estilo de aprendizaje y del tipo de tarea en los procesos de búsqueda en línea de estudiantes universitarios. Investig Bibl Arch Bibliotecol E Inf. 2015;29(65):115-35.

28. Hong S, Yu P. Comparison of the effectiveness of two styles of case-based learning implemented in lectures for developing nursing students' critical thinking ability: A randomized controlled trial. Int J Nurs Stud. marzo de 2017;68:16-24.

29. Van den Berg H. Changes in learning styles induced by practical training. Learn Individ Differ. 2015;40:84-9.

30. Montayre J, Sparks T. As I haven't seen a T-cell, video-streaming helps: Nursing students' preference towards online learning materials for biosciences. Rev Coll. 2017;1-6.

31. Torres PM. La práctica clínica: Un escenario para la enseñanza y el aprendizaje del cuidado del paciente con enfermedad crónica [Trabajo de Grado Maestría en Enfermería]. Universidad de Antioquia. Facultad de Enfermería.; 2017.

32. Stirling BV. Results of a study assessing teaching methods of faculty after measuring student learning style preference. Rev Nurse Educ Today. 2017;55:107-11.

33. Mentado Labao T, Medina Moya JL, Cruz Garcette L. Preparar para aprender: una manifestación del conocimiento didáctico del contenido en la práctica. Estud Sobre Educ. 2017;33:27-48.

34. Ramos Serpa G, López Falcón A, Echeverría E. Contribución de los meta-perfiles a mejorar la formación por competencias : el caso de la carrera de Enfermería en Contribution of meta-profiles to improve the competency-based training: the case of the Nursing career in UNIANDES. Rev Cienc Tecnol E Innov. 2016;3(2):135-49.

35. González Hernando C, Carbonero Martín MA, Lara Ortega F, Martín Villamor P. "Learning to learn" in Nursing Higher Education. Investig Educ En Enferm [Internet]. 12 de noviembre de 2013 [citado 8 de junio de 2018];31(3). Disponible en: https://aprendeenlinea.udea.edu.co/revistas/index.php/iee/ article/view/17508

36. Gimeno SJ. Comprender y Transformar la Enseñanza. 10a ed. España: Morata; 2008. 442.

37. Flórez Ochoa R. Evaluación pedagógica y cognición. Colombia: Mc Graw Hill; 2000. 226. 